\title{
Anomalous Josephson current, incipient time-reversal symmetry breaking, and Majorana bound states in interacting multi-level dots
}

\author{
Aldo Brunetti, ${ }^{1}$ Alex Zazunov, ${ }^{1}$ Arijit Kundu, ${ }^{2}$ and Reinhold Egger ${ }^{1}$ \\ 1 Institut für Theoretische Physik, Heinrich-Heine-Universität, D-40225 Düsseldorf, Germany \\ 2 Department of Physics, Indiana University, 727 East Third Street, Bloomington, IN 47405-7105 USA
}

(Dated: September 26, 2018)

\begin{abstract}
We study the combined effects of spin-orbit interaction, magnetic field, and Coulomb charging on the Josephson current-phase relation, $I(\varphi)$, for a multi-level quantum dot tunnel-contacted by two conventional $s$-wave superconductors with phase difference $\varphi$. A general model is formulated and analyzed in the cotunneling regime (weak tunnel coupling) and in the deep subgap limit, fully taking into account interaction effects. We determine the conditions for observing a finite anomalous supercurrent $I_{a}=I(\varphi=0)$. For a two-level dot with spin-orbit coupling and arbitrarily weak Zeeman field $B$, we find the onset behavior $I_{a} \propto \operatorname{sgn}(B)$ in the presence of interactions, suggesting the incipient spontaneous breakdown of time-reversal symmetry. We also provide conditions for realizing spatially separated (but topologically unprotected) Majorana bound states in a double dot variant of this system. Here Majoranas are predicted to leave a clear signature in the $2 \pi$-periodic current-phase relation.
\end{abstract}

PACS numbers: $74.50 .+\mathrm{r}, 74.45 .+\mathrm{c}, 74.78 . \mathrm{Na}$

\section{INTRODUCTION}

Studies of the current-phase relation $(\mathrm{CPR})$ in a Josephson junction, where a weak link connects two superconductors with phase difference $\varphi$, have provided ever new surprises over the past fifty years $\underline{\underline{1}}$ Nowadays, Josephson junctions showing novel and rich behavior can be formed by sandwiching a nanoscale conductor - collectively referred to as 'quantum dot' below, e.g., a semiconductor dot or nanowire, or a single molecule - between two superconductors $\stackrel{2,3}{=}$ The interest in such nanoscale hybrid devices has sharply increased recently due to technological advances, allowing to fabricate and manipulate well-characterized setups and raising the hope for new applications, as well as by the prospect of realizing Majorana fermions. To mention just a few key experiments, gate-tunable supercurrents through the twodimensional electron gas in semiconductors have been demonstrated,,$\underline{\underline{4}}-\underline{8}$ the CPR of superconducting atomic point contacts has been measured using a loop geometry,$\stackrel{9}{ }$ and the direct spectroscopy of Andreev bound states in carbon nanotube devices was reported $\stackrel{10}{\underline{10}}$ The phenomena studied below will be particularly pronounced for strong spin-orbit coupling (SOC) in the nanoscale conductor. Note that strong SOC is naturally present in InAs or InSb $\stackrel{11-20}{=}$ and in self-assembled SiGe quantum $\operatorname{dots} \stackrel{21}{2} \mathrm{SOC}$ is often responsible for nontrivial topological properties and the emergence of Majorana fermions in very similar settings $\stackrel{22-28}{-2}$ Majoranas have attracted wide attention after recent experiments reported first transport signatures such as those expected for Majorana fermions $29-32$

In this paper, we study a general model for the equilibrium Josephson current through a multi-level quantum dot tunnel-contacted by two conventional $s$-wave BCS superconductors with phase difference $\varphi$ and superconducting gap $\Delta$. Our dot Hamiltonian $H_{d}$, see Eq. (2.1) below, takes into account arbitrary SOC terms, magnetic (orbital and Zeeman) field effects, and Coulomb charging interactions. Moreover, the tunnel contacts are described by a general tunneling Hamiltonian $H_{t}$, see Eq. (2.7) below, allowing for inter-orbital phase shifts and asymmetric contacts. Our analysis is mostly devoted to two complementary regimes where analytical progress is possible, namely the cotunneling regime, realized for weak tunneling, and the deep subgap regime ('atomic limit'), where $\Delta$ represents the largest energy scale. We explore in detail the ground-state Josephson CPR, $I(\varphi)$, which can reveal two particularly interesting phenomena in such a setting, namely the anomalous Josephson effect and Majorana bound states (MBSs).

The anomalous Josephson effect is characterized by a finite supercurrent flowing at zero phase difference, $I_{a} \equiv I(\varphi=0) \neq 0 . \quad$ Comparing to the conventional Josephson relation, $I(\varphi)=I_{c} \sin \varphi$ with critical current $I_{c}$, this is equivalent to a $\varphi_{0}$ phase shift, i.e., $I_{a}=I_{c} \sin \varphi_{0}$. Junctions with $I_{a} \neq 0$ are thus commonly referred to as ' $\varphi_{0}$-junctions', where SOC is typically a crucial ingredient. The Josephson CPR for quantum dots with SOC has been studied in many theoretical works,$\underline{33}-\underline{44}$ and the conditions for $\varphi_{0}$-junction behavior have been clarified in the noninteracting case,$\underline{34,35,39}-\underline{41} \mathrm{In}$ contrast to the widely known 0 - and $\pi$-junctions, $\frac{1}{,}$ where $\varphi_{0}=0$ and $\varphi_{0}=\pi$, respectively, a general $\varphi_{0}$-junction can have direction-dependent critical currents, $\stackrel{39,43}{=}$ i.e., $I_{c 1}=\max [I(\varphi)]$ and $I_{c 2}=\max [-I(\varphi)]$ are different. The $\varphi_{0}$-junction can thus act as a phase battery $\underline{45}$ or as superconducting rectifier, $\stackrel{39,43}{=}$ promising novel device applications. While it is well-established ${ }^{45-49}$ and also experimentally observed ${ }^{50}$ that spin-active interfaces, e.g., for a ferromagnetic 'dot' region, allow one to realize a $\varphi_{0}$-junction, we here focus on semiconducting or molecular systems with spin-conserving and spin-independent interfaces, where $\varphi_{0}$-junction behavior is quite nontriv- 
ial. $\varphi_{0}$-junctions were also predicted but never observe in unconventional superconductors $51-55$

So far, the necessary conditions for anomalous supercurrents have only been determined for noninteracting dots, where one needs finite SOC and a suitably oriented magnetic field. In addition, asymmetric tunnel contacts with non-commuting hybridization matrices, $\Gamma^{(L)} \neq \Gamma^{(R)}$, are required. This imposes a chirality condition which is necessary to have $I_{a} \neq 0$, see Ref. 41 and Sec. III below. We find that the Coulomb charging energy $E_{c}$ does not change these necessary conditions, but it can be responsible for a dramatic enhancement of the anomalous supercurrent. The most interesting enhancement is related to an interaction-induced behavior with $I_{a} \propto \operatorname{sgn}(B)$ for arbitrarily weak time-reversal symmetry (TRS) breaking field $B$. Such a behavior suggests that TRS is spontaneously broken. However, thermal fluctuations can suppress $I_{a}$, and we therefore interpret this enhancement of $I_{a}$ compared to the usual noninteracting behavior, $\stackrel{41}{,} I_{a}(B \rightarrow 0) \propto B$, as 'incipient' spontaneously broken TRS. This effect generally happens whenever two $B=0$ Kramer's partner states contribute with opposite sign to $I_{a}$. A small magnetic field then lifts the degeneracy, while the Coulomb interactions create a gap and effectively project away the higher energy state. As a consequence, interaction-induced enhancement is especially pronounced for small $B$ and if $E_{c}$ exceeds all other energy scales of interest. Concrete parameter regimes where this effect occurs will be discussed in Sec. IV. We mention in passing that spontaneously broken TRS was also reported in a recent mean-field study ${ }^{55}$ for a singlelevel Anderson dot between a two-band $\left(s_{ \pm}\right)$and a singleband ( $s$-wave) superconductor. However, this effect can be traced back to phase frustration 55 and strongly differs from our scenario. Technically related works have also studied the supercurrent in the cotunneling regime for dots coupled to a local phonon mode ${ }^{56}$ and to a twolevel system. $\frac{57}{2}$ Other studies of the Josephson effect for interacting double dots have either disregarded SOC $58-60$ or did not address the phenomena investigated here 61

Besides analyzing the anomalous supercurrent, in Sec. $\mathrm{V}$ we also address the possibility of MBS formation in an interacting double dot with SOC and Zeeman field. The double dot is contained as special case in our general multi-level Hamiltonian, and our theory is directly applicable to such a two-orbital case with well separated orbitals. Majorana fermions are emergent quasiparticles that equal their own antiparticle. They are of much interest in the context of topological quantum computation. ${ }^{22-26}$ When our 'dot' region corresponds to a semiconductor nanowire, one effectively can realize Kitaev's chain model which (in the right parameter regime) allows for a pair of topologically protected MBSs localized near the nanowire ends ${ }^{27,28}$ ('Topological protection' implies that small parameter changes not closing a bulk gap cannot remove the MBSs.) As discussed by Lejinse and Flensberg,, 62 see also Refs. 63,64, a simpler variant, albeit with topologically unprotected Majorana fermions, can be realized for two Coulomb-blockaded single-level dots coupled to a superconductor. Similarly, in our setting a pair of spatially separated MBSs can also be realized. Remarkably, these Majoranas could be detected through the highly unusual features in the $2 \pi$ periodic CPR described below.

The structure of the remainder of this article is as follows. In Sec. III, a general model for the S-Dot-S hybrid structure is introduced. We allow for arbitrary singleparticle Hamiltonians in the dot region, and take into account Coulomb charging effects. Integrating out the noninteracting fermions in the superconducting electrodes, we arrive at an effective partition function expressed in terms of dot variables only, which then allows to extract the Josephson CPR by a phase derivative. For concrete results, we employ a generic two-orbital dot with a Zeeman field and (Rashba or Dresselhaus) SOC. In Sec. III, we discuss the two approaches used in this work. First, we study the cotunneling regime by perturbation theory in the tunnel couplings. The general ground-state CPR is derived, see Eq. (3.1), with $I_{a}$ expressed in terms of matrices $J$ and $Q$, see Eq. (3.4). $J$ depends only on single-particle quantities and imposes necessary conditions for $I_{a} \neq 0$, while $Q$ encapsulates interaction effects. As second approach, we study the 'atomic limit', $\Delta \rightarrow \infty$, where the proximity effect of the superconducting leads is contained in an effective dot Hamiltonian. In Sec. IV we address the anomalous Josephson effect for a two-level dot, and in Sec. V] we show that a pair of spatially separated MBSs emerges for suitably chosen parameters in a double dot device. Finally, we offer some concluding remarks in Sec. VI. We often use units with $\hbar=e=k_{B}=1$.

\section{MODEL AND EFFECTIVE PARTITION FUNCTION}

\section{A. General model}

We study a general model describing the Josephson effect in a large variety of interacting nanostructures, where a central region ('dot') is tunnel-coupled to two conventional $s$-wave superconducting leads, $H=H_{d}+H_{t}+H_{l}$. Following standard arguments, 65 we take into account Coulomb interactions, SOC, and magnetic field effects only on the dot, but not in the bulk electrodes nor in the tunnel contact. For $M$ relevant (spin-degenerate) electronic orbitals in the central dot region, the dot Hamiltonian is taken in the form

$$
H_{d}=\sum_{n \sigma, n^{\prime} \sigma^{\prime}} d_{n \sigma}^{\dagger} h_{n \sigma, n^{\prime} \sigma^{\prime}} d_{n^{\prime} \sigma^{\prime}}+E_{c}\left(\hat{N}-n_{g}\right)^{2}
$$

where the operator $d_{n \sigma}^{\dagger}$ creates a dot electron in a singleparticle state with orbital quantum number $n=1, \ldots, M$ and spin projection $\sigma=\uparrow, \downarrow$. The $2 M \times 2 M$ Hermitian matrix $h_{n \sigma, n^{\prime} \sigma^{\prime}}$ encapsulates the single-particle content, including SOC and magnetic field effects. At this 
stage, we make no assumptions about the SOC, allowing for rather general statements regarding the anomalous Josephson effect. Importantly, the $h$ matrix can always be diagonalized by a unitary transformation, $U^{\dagger} h U=\operatorname{diag}\left(E_{\nu}\right)$, with the single-particle energies $E_{\nu}$ $(\nu=1, \ldots, 2 M)$. We then have associated fermionic operators, $c_{\nu}$, with

$$
d_{n \sigma}=\sum_{\nu=1}^{2 M} U_{n \sigma, \nu} c_{\nu}
$$

which correspond to single-particle eigenstates of the isolated dot. The $d_{n \sigma}$ operators instead will be taken to represent dot fermion modes tunnel-coupled to the leads. Both representations are, of course, equivalent, and the benefits of using the $c_{\nu}$ should become clear below. After the unitary transformation,

$$
H_{d}=\sum_{\nu} E_{\nu} c_{\nu}^{\dagger} c_{\nu}+E_{c}\left(\hat{N}-n_{g}\right)^{2} .
$$

The capacitive Coulomb charging term is only sensitive to the total dot fermion number operator,

$$
\hat{N}=\sum_{n \sigma} d_{n \sigma}^{\dagger} d_{n \sigma}=\sum_{\nu} c_{\nu}^{\dagger} c_{\nu}
$$

where the charging energy, $E_{c}$, sets the energy cost for adding or removing electrons. The real number $n_{g}$ is proportional to a backgate voltage and regulates the average number of electrons on the dot. It is worth mentioning that the above charging term generically describes the dominant interaction contribution .65 For later use, we also define the Coulomb energy differences $W_{k}$ (integer $k)$,

$$
W_{k}=E_{c}\left(N_{0}+k-n_{g}\right)^{2}-E_{c}\left(N_{0}-n_{g}\right)^{2},
$$

where the integer $N_{0}$ denotes the ground-state electron number on the dot.

The left and right $(j=L, R)$ superconducting leads are described by standard bulk BCS Hamiltonians. For simplicity, we assume that they have identical gap $\Delta$ and normal-state dispersion relation $\xi_{\mathbf{k}}$, with chemical potential $\mu_{S}=0 . \underline{66}$ Moreover, we use a gauge where the order parameter phases appear in the tunneling Hamiltonian $H_{t}$ only, and $\Delta \geq 0$ is real-valued. It is then convenient to switch to particle-hole (Nambu) space and introduce the spinor $\Psi_{j \mathbf{k}}=\left(c_{j, \mathbf{k}, \uparrow}, c_{j,-\mathbf{k}, \downarrow}^{\dagger}\right)^{T}$, where $c_{j, \mathbf{k}, \sigma}^{\dagger}$ creates an electron in lead $j$ with momentum $\mathbf{k}$ and spin projection $\sigma$. The lead Hamiltonian is then given by

$$
H_{l}=\sum_{j=L, R} \sum_{\mathbf{k}} \Psi_{j \mathbf{k}}^{\dagger}\left(\begin{array}{cc}
\xi_{\mathbf{k}} & \Delta \\
\Delta & -\xi_{\mathbf{k}}
\end{array}\right) \Psi_{j \mathbf{k}} .
$$

Finally, we come to $H_{t}$, where a complex-valued tunneling matrix element $t_{j, \mathbf{k}, \sigma ; n, \sigma^{\prime}}$ gives the probability amplitude for transfer of an electron from dot state $\left(n, \sigma^{\prime}\right)$ to lead state $(j, \mathbf{k}, \sigma)$. To simplify the analysis, we adopt the standard wide-band approximation for the leads ${ }^{65}$ and neglect the $\mathbf{k}$-dependence of the tunneling matrix elements. Leaving aside spin-active interfaces, tunneling is assumed to be spin-conserving and spin-independent, $t_{j, \mathbf{k}, \sigma ; n \sigma^{\prime}}=\delta_{\sigma \sigma^{\prime}} t_{j, n}$, and $H_{t}$ is determined by $2 M$ complex-valued parameters $t_{j, n}$. Employing the Nambu spinor notation also for the dot fermions, $D_{n}=\left(d_{n, \uparrow}, d_{n, \downarrow}^{\dagger}\right)^{T}$, we obtain

$$
\begin{aligned}
H_{t} & =\sum_{j=L, R} \sum_{\mathbf{k}} \sum_{n=1}^{M} \Psi_{j \mathbf{k}}^{\dagger} T_{j, n} D_{n}+\text { H.c. } \\
T_{j, n} & =\left(\begin{array}{cc}
e^{i \phi_{j} / 2} t_{j, n} & 0 \\
0 & -e^{-i \phi_{j} / 2} t_{j, n}^{*}
\end{array}\right),
\end{aligned}
$$

where $\phi_{j}$ is the superconducting phase in lead $j$.

\section{B. Current-phase relation}

In this paper, we study the equilibrium Josephson CPR in the zero-temperature limit, $T \rightarrow 0$. A formally exact expression for the $\mathrm{CPR}$ can be obtained from the partition function, $Z=\operatorname{Tr} e^{-\beta H}$, with $\beta=1 / T$. We start by employing Wick's theorem to trace out the noninteracting lead fermions. In the interaction picture, let $H_{0}=H-H_{t}$ govern the imaginary-time $(\tau)$ evolution. For arbitrary operator $\mathcal{O}$, we use the notation 67

$$
\mathcal{O}(\tau)=e^{H_{0} \tau} \mathcal{O} e^{-H_{0} \tau}, \quad \overline{\mathcal{O}}(\tau)=e^{H_{0} \tau} \mathcal{O}^{\dagger} e^{-H_{0} \tau} .
$$

The partition function then reads

$$
\begin{aligned}
Z & =\operatorname{Tr}_{d} \operatorname{Tr}_{l}\left(e^{-\beta H_{0}} \mathcal{T} e^{-\int_{0}^{\beta} d \tau H_{t}(\tau)}\right) \\
& =Z_{l} \operatorname{Tr}_{d}\left(e^{-\beta H_{d}} \mathcal{T} e^{-S_{t}}\right)
\end{aligned}
$$

where $\mathcal{T}$ denotes time ordering. The traces $\operatorname{Tr}_{d, l}$ are over dot and lead Hilbert spaces, respectively, with $Z_{l}=$ $\operatorname{Tr}_{l} e^{-\beta H_{l}}$. In Eq. (2.9), we have averaged over the leads, and using $\left\langle H_{t}(\tau)\right\rangle_{l}=Z_{l}^{-1} \operatorname{Tr}_{l}\left[e^{-\beta H_{l}} H_{t}(\tau)\right]=0$, Wick's theorem implies that $S_{t}$ in Eq. (2.9) is completely determined by the Gaussian correlator

$$
S_{t}=-\frac{1}{2} \int_{0}^{\beta} d \tau d \tau^{\prime}\left\langle\mathcal{T} H_{t}(\tau) H_{t}\left(\tau^{\prime}\right)\right\rangle_{l}
$$

Inserting $H_{t}$ [Eq. (2.7)], we obtain

$$
S_{t}=\frac{1}{2} \int_{0}^{\beta} d \tau d \tau^{\prime} \sum_{n n^{\prime}} \bar{D}_{n}(\tau) \Lambda_{n n^{\prime}}\left(\tau-\tau^{\prime}\right) D_{n^{\prime}}\left(\tau^{\prime}\right)
$$

where $\Lambda_{n n^{\prime}}\left(\tau-\tau^{\prime}\right)=2 \sum_{j} T_{j, n}^{\dagger} G_{l}\left(\tau-\tau^{\prime}\right) T_{j, n^{\prime}}$ is expressed in terms of the lead Green's function,

$$
\begin{aligned}
G_{l}\left(\tau-\tau^{\prime}\right) & =-\sum_{\mathbf{k}}\left\langle\mathcal{T} \Psi_{j \mathbf{k}}(\tau) \bar{\Psi}_{j \mathbf{k}}\left(\tau^{\prime}\right)\right\rangle_{l} \\
& =-\pi \nu_{0} T \sum_{m} \frac{e^{-i \omega_{m}\left(\tau-\tau^{\prime}\right)}}{\sqrt{\omega_{m}^{2}+\Delta^{2}}}\left(\begin{array}{cc}
i \omega_{m} & \Delta \\
\Delta & i \omega_{m}
\end{array}\right)
\end{aligned}
$$


which is identical for both leads. Here we have employed the wide-band approximation, with normal-state lead density of states $\nu_{0}=\sum_{\mathbf{k}} \delta\left(\xi_{\mathbf{k}}\right)$, and fermion Matsubara frequencies $\omega_{m}=\pi T(2 m+1)$ (integer $m$ ). The kernel $\Lambda$ in Eq. (2.11), describing the effects of the tracedout leads on the dot fermions, thus reads

$$
\Lambda_{n n^{\prime}}(\tau)=\sum_{j=L, R} \Gamma_{n n^{\prime}}^{(j)}\left(\begin{array}{cc}
\partial_{\tau} & \Delta e^{-i \phi_{j}} \\
\Delta e^{i \phi_{j}} & \partial_{\tau}
\end{array}\right) f(\tau),
$$

where the tunnel contacts are described by Hermitian $M \times M$ hybridization matrices,

$$
\Gamma_{n n^{\prime}}^{(j)}=2 \pi \nu_{0} t_{j, n}^{*} t_{j, n^{\prime}},
$$

and we use the auxiliary function

$$
f(\tau)=T \sum_{m} \frac{e^{-i \omega_{m} \tau}}{\sqrt{\omega_{m}^{2}+\Delta^{2}}} .
$$

Notice that $\Lambda$ factorizes in orbital and Nambu subspaces.

The Josephson current flowing through contact $j$ to the dot follows from the ground-state average $\mathrm{e}^{\underline{\underline{1}}}$

$$
I_{j}=\frac{2 e}{\hbar} \partial_{\phi_{j}} F,
$$

where $F=-T \ln Z$ is the free energy. Current conservation dictates $I_{L, R}= \pm I(\varphi)$, where $\varphi=\phi_{L}-\phi_{R}$ is the gauge-invariant phase difference. Using Eqs. (2.9) and (2.16), the $T=0$ CPR, $I(\varphi)$, will be computed in Sec. III for the cotunneling regime and in the atomic limit.

\section{Two orbital levels}

For concrete results, we will consider a generic model with $M=2$ dot orbital levels, which provides a minimal setting for studying SOC effects, the anomalous supercurrent, and Majorana fermions. The $4 \times 4$ matrix $h$ describing the single-particle spectrum of the dot Hamiltonian $H_{d}$ [Eq. [2.1)] is taken in the generic form

$h=\left(\mu \tau_{0}+\epsilon \tau_{z}\right) \sigma_{0}+B \tau_{0} \sigma_{z}+\alpha \tau_{y}\left[\cos (\chi) \sigma_{z}+\sin (\chi) \sigma_{y}\right]$,

where $\tau_{x, y, z}\left(\sigma_{x, y, z}\right)$ are Pauli matrices in orbital (spin) space; the respective unity matrices are $\tau_{0}\left(\sigma_{0}\right)$. The physics is here determined by the interplay of a Rashbatype $\mathrm{SOC}$, whose strength is parameterized by the energy scale $\alpha$, and the magnetic Zeeman field, with energy scale $B$. In Eq. (2.17), $0 \leq \chi \leq \pi$ denotes the angle between the effective spin-orbit field and the Zeeman field. The bare $[\alpha=B=0]$ dot levels are $\mu \pm \epsilon$. For the specific 2D dot model studied in Ref. 37, it is straightforward to explicitly determine the model parameters entering Eq. (2.17).

Next we express the $2 \times 2$ (in orbital space) hybridization matrices [Eq. (2.14)] in the form

$$
\Gamma^{(j=L, R)}=\gamma_{j}\left(\begin{array}{cc}
e^{\lambda_{j}} & e^{i \delta_{j}} \\
e^{-i \delta_{j}} & e^{-\lambda_{j}}
\end{array}\right)
$$

where $\gamma_{j} \geq 0$ gives the overall hybridization strength of the respective contact, $\lambda_{j}$ parametrizes the orbital asymmetry (for $\lambda_{j}=0$, both orbitals couple symmetrically to the $j$ th lead), and $\delta_{j}$ is an inter-orbital phase shift. Since $\delta_{L, R}$ is independent of spin, these phase shifts have nothing to do with SOC. For instance, they could be caused by orbital magnetic fields; for the dot model proposed in Ref. 37, this follows by virtue of a gauge transformation transferring the orbital field dependence to the tunneling Hamiltonian. The phases $\delta_{L, R}$ may also be influenced by the dot geometry, in particular by contact asymmetries. It is worth stressing that for $\alpha \neq 0$ and $\Delta \neq 0$, one cannot gauge away the resulting phases $\delta_{L, R}$. For further convenience, we define the relative inter-orbital phase shift

$$
\delta=\delta_{L}-\delta_{R}
$$

In the absence of SOC, i.e., for $\alpha=0$, the dot Hamiltonian is diagonal in orbital space, and then only the phase difference $\delta$ cannot be gauged away.

We note that our assumption of $\mathbf{k}$-independent tunneling matrix elements implies that the phase shifts $\delta_{j}$ are also momentum-independent. If this assumption is violated, the $\delta_{j}$ are best treated as statistical variables. The resulting average may suppress $I_{a}$ while leaving critical currents basically unaffected. Since such generalizations are straightforward to implement, we here proceed by assuming $\mathbf{k}$-independent phase shifts $\delta_{L, R}$.

\section{JOSEPHSON CURRENT}

In this work, we compute the Josephson current for the above model using two complementary vantage points, namely by perturbation theory in the cotunneling regime and by employing an effective Hamiltonian valid in the deep subgap regime.

\section{A. Cotunneling regime}

The cotunneling regime is realized when all eigenvalues of the hybridization matrices $\Gamma^{(L, R)}$ are small against $\Delta$. In that case, perturbation theory in these Hermitian matrices is well-defined and allows for progress $\underline{68}$ Since $S_{t} \propto \Gamma^{(L, R)}$, see Eqs. (2.11) and (2.13), the free energy $F$ can be directly expanded in powers of $S_{t}$. Starting from Eq. (2.9) and using $\partial_{\phi_{j}}\left\langle S_{t}\right\rangle=0$, the lowest-order contribution to the Josephson current (2.16) is of order $\Gamma^{L} \Gamma^{R}$ and reads $I_{j}=-2 T\left\langle S_{t} \partial_{\phi_{j}} S_{t}\right\rangle$, where $\langle\cdots\rangle$ denotes the ground-state expectation value for the closed dot Hamiltonian $H_{d}$. Inserting Eq. (2.11), we find $I_{L, R}= \pm I(\varphi)$, in accordance with current conservation, where

$$
I(\varphi)=I_{0} \sin \varphi+I_{a} \cos \varphi
$$


with the currents

$$
\begin{aligned}
\left(\begin{array}{c}
I_{0} \\
i I_{a}
\end{array}\right) & =\sum_{n m n^{\prime} m^{\prime}}\left(\begin{array}{c}
\Gamma_{n m}^{(L)} \Gamma_{n^{\prime} m^{\prime}}^{(R)}+(L \leftrightarrow R) \\
\Gamma_{n m}^{(L)} \Gamma_{n^{\prime} m^{\prime}}^{(R)}-(L \leftrightarrow R)
\end{array}\right) \\
& \times \frac{\Delta^{2}}{2 \beta} \int_{0}^{\beta} d \tau_{1} d \tau_{2} d \tau_{1}^{\prime} d \tau_{2}^{\prime} f\left(\tau_{1}-\tau_{2}\right) f\left(\tau_{1}^{\prime}-\tau_{2}^{\prime}\right) \\
& \times\left\langle\mathcal{T} d_{n \downarrow}\left(\tau_{1}\right) d_{m \uparrow}\left(\tau_{2}\right) \bar{d}_{n^{\prime} \uparrow}\left(\tau_{1}^{\prime}\right) \bar{d}_{m^{\prime} \downarrow}\left(\tau_{2}^{\prime}\right)\right\rangle .
\end{aligned}
$$

The critical current is $I_{c}=\sqrt{I_{0}^{2}+I_{a}^{2}}$, where we find $I_{c 1}=I_{c 2}=I_{c}$ in the cotunneling regime. It is now crucial to use the unitary transformation $U$ in Eq. (2.2) to switch from the $d_{n \sigma}$ to the $c_{\nu}$ fermions. The latter represent the eigenstates of the isolated interacting dot. Using $f(\tau)=f(-\tau)$, we observe that only the antisymmetric part of the transformed hybridization matrices enters the expressions for $I_{0, a}$. In terms of the antisymmetric $2 M \times 2 M$ matrices

$$
\tilde{\Gamma}_{\nu \mu}^{(j=L, R)}=\sum_{n m} \Gamma_{n m}^{(j)}\left(U_{n \downarrow, \nu} U_{m \uparrow, \mu}-U_{n \downarrow, \mu} U_{m \uparrow, \nu}\right),
$$

we find from Eq. (3.2) for the anomalous Josephson current

$$
I_{a}=\frac{e \Delta^{2}}{\hbar} \sum_{\nu>\mu} J_{\nu \mu} Q_{\nu \mu}
$$

with the symmetric $2 M \times 2 M$ matrices

$$
\begin{gathered}
J_{\nu \mu}=\operatorname{Im}\left(\tilde{\Gamma}_{\nu \mu}^{(L)}\left[\tilde{\Gamma}^{(R)}\right]_{\nu \mu}^{*}\right) \\
Q_{\nu \mu}=-T \int_{0}^{\beta} d \tau_{1} d \tau_{2} d \tau_{1}^{\prime} d \tau_{2}^{\prime} f\left(\tau_{1}-\tau_{2}\right) f\left(\tau_{1}^{\prime}-\tau_{2}^{\prime}\right) \\
\times\left\langle\mathcal{T} c_{\nu}\left(\tau_{1}\right) c_{\mu}\left(\tau_{2}\right) \bar{c}_{\nu}\left(\tau_{1}^{\prime}\right) \bar{c}_{\mu}\left(\tau_{2}^{\prime}\right)\right\rangle
\end{gathered}
$$

The current $I_{0}$ follows in similar form,

$$
I_{0}=\frac{e \Delta^{2}}{\hbar} \sum_{\nu>\mu} \operatorname{Re}\left(\tilde{\Gamma}_{\nu \mu}^{(L)}\left[\tilde{\Gamma}_{\nu \mu}^{(R)}\right]^{*}\right) Q_{\nu \mu}
$$

We can now use Eq. 3.4 to infer general conditions for the anomalous Josephson effect to exist within the cotunneling regime. As necessary condition for $I_{a} \neq 0$, we observe that $J_{\nu \mu} \neq 0$ must be satisfied for at least one index pair $\nu>\mu$. Note that $J_{\nu \mu}$ depends only on single-particle quantities, such as tunneling matrix elements, SOC, and Zeeman fields. The role of interactions is encoded in the $Q$ matrix and can be crucial in breaking the balance between time-reversed processes, which may then induce the anomalous Josephson effect. Note that this condition is very general and holds for arbitrary matrices $h$ determining the single-particle spectrum.

It is interesting to see what happens for a single-level dot, $M=1$, where $\Gamma^{(L)}$ and $\Gamma^{(R)}$ are just real numbers. The antisymmetric $\tilde{\Gamma}^{(L, R)}$ matrices in Eq. (3.3) are then fully determined by $\tilde{\Gamma}_{21}^{(j)}=\Gamma^{(j)}\left(U_{\downarrow, 2} U_{\uparrow, 1}-U_{\downarrow, 1} U_{\uparrow, 2}\right)$, which immediately yields $J=0$ in Eq. 3.5. Hence no anomalous Josephson current is possible in a singleorbital dot, even when interactions are included. A minimal model for this effect has to start from $M=2$ orbital dot levels, see Sec. IV] where we study the conditions for the anomalous Josephson effect in a concrete and experimentally relevant setting.

General conditions (beyond the cotunneling regime) for the anomalous Josephson effect can also be deduced directly from symmetry considerations. We exemplify this here by analyzing the supercurrent through an inversion-symmetric two-dimensional dot with in-plane (purely Zeeman) magnetic field $B$ and SOC strength $\alpha$. A spatial inversion operation, $(x, y) \rightarrow(-x,-y)$, is implemented by (i) exchanging the lead indices, $L \leftrightarrow R$, (ii) inverting the phase difference, $\varphi \rightarrow-\varphi$, (iii) changing the sign of the SOC, $\alpha \rightarrow-\alpha$, and (iv) also changing the sign of the (in-plane) Zeeman field, $B \rightarrow-B$. Since $I(\varphi) \rightarrow-I(-\varphi)$ under spatial inversion, Eq. 3.1 implies that the anomalous supercurrent must satisfy the symmetry relation

$$
I_{a}\left(\Gamma^{(L)}, \Gamma^{(R)}, B, \alpha\right)=-I_{a}\left(\Gamma^{(R)}, \Gamma^{(L)},-B,-\alpha\right) .
$$

Similarly, we deduce an additional condition from the supercurrent behavior under a time reversal operation,

$$
I_{a}\left(\Gamma^{(L)}, \Gamma^{(R)}, B, \alpha\right)=-I_{a}\left(\Gamma^{(L)}, \Gamma^{(R)},-B, \alpha\right)
$$

which implies that $I_{a}$ is always odd in $B$.

Let us next address the $Q$ matrix in Eq. (3.6), which only depends on properties of the closed dot. In the cotunneling regime, interactions can affect the CPR only through this matrix. In general, $4 !=24$ terms involving all possible permutations of time-ordered fermion operators will be generated from Eq. (3.6). However, if the closed dot has a non-degenerate interacting ground state $|G\rangle$, Eq. (3.6) allows for simplifications in the $\beta \rightarrow \infty$ limit of interest here. Excluding 'accidental' degeneracies, this step assumes that a TRS-breaking magnetic field is present. Effectively, only three permutations in Eq. (3.6) are relevant and $Q_{\nu \mu}$ can be expressed in terms of the three real-valued functions

$$
\begin{aligned}
\mathcal{Q}_{i}\left(\epsilon_{a}, \epsilon_{b}, \epsilon_{c}\right) & =\frac{1}{\beta} \int_{0}^{\beta} d \tau_{a} \int_{0}^{\tau_{a}} d \tau_{b} \int_{0}^{\tau_{b}} d \tau_{c} \int_{0}^{\tau_{c}} d \tau_{d} \\
& \times e^{-\epsilon_{a}\left(\tau_{a}-\tau_{b}\right)-\epsilon_{b}\left(\tau_{b}-\tau_{c}\right)-\epsilon_{c}\left(\tau_{c}-\tau_{d}\right)}(3.10), i=1, \\
& \times\left\{\begin{array}{l}
f\left(\tau_{a}-\tau_{b}\right) f\left(\tau_{c}-\tau_{d}\right), i=2, \\
f\left(\tau_{a}-\tau_{d}\right) f\left(\tau_{b}-\tau_{c}\right), i=2, \\
f\left(\tau_{a}-\tau_{c}\right) f\left(\tau_{b}-\tau_{d}\right), i=3,
\end{array}\right.
\end{aligned}
$$

where $\epsilon_{a, b, c} \geq 0$ are possible excitation energies. Switching to the frequency domain and using Eq. (2.15), we 
obtain 69

$$
\begin{aligned}
& \mathcal{Q}_{i}=\int \frac{d \omega_{1} d \omega_{2}}{(2 \pi)^{2}} \frac{1}{\sqrt{\left(\omega_{1}^{2}+\Delta^{2}\right)\left(\omega_{2}^{2}+\Delta^{2}\right)}} \\
& \times \begin{cases}\left(1-\delta_{\epsilon_{b}, 0}\right) /\left[\left(i \omega_{1}+\epsilon_{a}\right)\left(i \omega_{2}+\epsilon_{c}\right) \epsilon_{b}\right], & i=1, \\
1 /\left[\left(i \omega_{1}+\epsilon_{a}\right)\left(i \omega_{1}+\epsilon_{c}\right)\left(i \omega_{1}+i \omega_{2}+\epsilon_{b}\right)\right], & i=2, \\
1 /\left[\left(i \omega_{1}+\epsilon_{a}\right)\left(i \omega_{2}+\epsilon_{c}\right)\left(i \omega_{1}+i \omega_{2}+\epsilon_{b}\right)\right], & i=3 .\end{cases}
\end{aligned}
$$

Notice that the $\mathcal{Q}_{i}$ are invariant under the exchange $\epsilon_{a} \leftrightarrow \epsilon_{c}$. Consider now the ground state $|G\rangle$ of the closed dot Hamiltonian $H_{d}$ in Eq. (2.3), with $N_{0}$ electrons on the dot, $\hat{N}|G\rangle=N_{0}|G\rangle$. Assuming that $|G\rangle$ is nondegenerate, the filling factor $n_{\nu}$ for each single-particle state $\nu=1, \ldots, 2 M$ is known. Arranging the $E_{\nu}$ as ordered sequence, $E_{1} \leq E_{2} \leq \cdots \leq E_{2 M}$, the result is

$$
n_{\nu}=\left\langle G\left|c_{\nu}^{\dagger} c_{\nu}\right| G\right\rangle= \begin{cases}1, & \nu \leq N_{0} \\ 0, & \nu>N_{0}\end{cases}
$$

For given index pair $\nu>\mu$, three possibilities arise, namely $\left(n_{\nu}, n_{\mu}\right)=(0,0),(1,1)$, and $(0,1)$. It is then straightforward to determine the excitation energies $\epsilon_{a, b, c}$ by comparing Eqs. (3.6) and (3.10) in those three cases. To state the final result for $Q$, it is useful to introduce the positive energies

$$
\begin{aligned}
\tilde{E}_{\nu} & =\left(1-2 n_{\nu}\right) E_{\nu}+W_{1-2 n_{\nu}}, \\
\tilde{E}_{\nu \mu} & =\left(1-2 n_{\nu}\right) E_{\nu}+\left(1-2 n_{\mu}\right) E_{\mu}+W_{2-2 n_{\nu}-2 n_{\mu}},
\end{aligned}
$$

with the Coulomb energy differences $W_{k}$ in Eq. (2.5). (Note that for $E_{c}=0$, we have $1-2 n_{\nu}=\operatorname{sgn}\left(E_{\nu}\right)$ and hence $\tilde{E}_{\nu}=\left|E_{\nu}\right|$.) We then obtain the symmetric $Q$ matrix,

$$
\begin{aligned}
Q_{\nu \mu} & =\left(1-2 n_{\nu}\right)\left(1-2 n_{\mu}\right)\left[2 \mathcal{Q}_{i_{1}}\left(\tilde{E}_{\nu}, \tilde{E}_{\nu \mu}, \tilde{E}_{\mu}\right)\right. \\
& +\mathcal{Q}_{i_{2}}\left(\tilde{E}_{\nu}, \tilde{E}_{\nu \mu}, \tilde{E}_{\nu}\right) \\
& \left.+\mathcal{Q}_{i_{2}}\left(\tilde{E}_{\mu}, \tilde{E}_{\nu \mu}, \tilde{E}_{\mu}\right)+2 \mathcal{Q}_{i_{3}}\left(\tilde{E}_{\nu}, 0, \tilde{E}_{\mu}\right)\right]
\end{aligned}
$$

where the indices are $i_{1}=i_{2}=1$ and $i_{3}=3$ for $n_{\nu}=n_{\mu}$. For $n_{\nu} \neq n_{\mu}$, we instead have $i_{1}=i_{3}=2$ and $i_{2}=3$.

We proceed by discussing the limit of strong Coulomb blockade. For $E_{c} \rightarrow \infty$, the cotunneling supercurrent is generally strongly suppressed. Technically, this suppression can be seen from Eq. (3.11): all excitation energies scale as $\epsilon_{a, b, c} \propto E_{c} \rightarrow \infty$, which implies $Q_{\nu \mu} \rightarrow 0$ and thus $I_{0, a} \rightarrow 0$. This argument only breaks down for halfinteger values of $n_{g}$, where the strong charging term in $H_{d}$ allows for two degenerate charge states with particle numbers $N_{0}=N_{0, \pm} \equiv n_{g} \pm 1 / 2$. Let us therefore now focus on half-integer values of $n_{g}$, where the single-particle spectrum, $\left\{E_{\nu}\right\}$, ultimately determines the ground state and, in particular, which particle number $N_{0}$ is realized (either $N_{0,+}$ or $N_{0,-}$ ). Using that for $N_{0}=N_{0, \pm}$, we have the Coulomb energy difference $W_{\mp 1}=0$, Eq. (3.14) simplifies to

$$
\begin{aligned}
& Q_{\nu \mu}^{\left(N_{0,+}\right)}=2 n_{\nu} n_{\mu} \mathcal{Q}_{3}\left(-E_{\nu}, 0,-E_{\mu}\right) \\
& -\left[\left(1-n_{\nu}\right) n_{\mu} \mathcal{Q}_{3}\left(-E_{\mu}, E_{\nu}-E_{\mu},-E_{\mu}\right)+(\nu \leftrightarrow \mu)\right], \\
& Q_{\nu \mu}^{\left(N_{0,-}\right)}=2\left(1-n_{\nu}\right)\left(1-n_{\mu}\right) \mathcal{Q}_{3}\left(E_{\nu}, 0, E_{\mu}\right) \\
& -\left[\left(1-n_{\nu}\right) n_{\mu} \mathcal{Q}_{3}\left(E_{\nu}, E_{\nu}-E_{\mu}, E_{\nu}\right)+(\nu \leftrightarrow \mu)\right] .
\end{aligned}
$$

It is instructive to examine Eq. (3.15) for a spindegenerate single-level $(M=1)$ dot without SOC and without magnetic field. Both single-particle states $(\nu=\uparrow$, $\downarrow$ ) then have identical energy, say $E_{\nu}=x \Delta$ with some dimensionless parameter $x$, and Eq. (3.15) yields ${ }^{70}$

$$
Q_{\uparrow \downarrow}=\mathcal{Q}_{3}(|x| \Delta, 0,|x| \Delta) \times \begin{cases}2, & N_{0}=0,2, \\ -1, & N_{0}=1,\end{cases}
$$

where Eq. (3.11) gives $(x>0)$

$$
\mathcal{Q}_{3}(x \Delta, 0, x \Delta)=\frac{1}{\pi^{2} \Delta^{3}} \frac{(\pi / 2)^{2}(1-x)-\operatorname{Arccos}^{2} x}{x\left(1-x^{2}\right)} .
$$

Noting that $I_{a}=0$ for $M=1$, the critical current $I_{c}$ directly follows from Eq. (3.7), where Eq. (3.16) predicts $\pi$-junction [0-junction] behavior, with $I(\varphi)=-I_{c} \sin \varphi$ $\left[I(\varphi)=I_{c} \sin \varphi\right]$, for $N_{0}=1\left[N_{0}=0,2\right]$. We have thereby reproduced well-known results. $\frac{1,3}{1}$ In general, in the strong Coulomb blockade limit $E_{c} \rightarrow \infty$, we find $\pi$ junction behavior for odd $N_{0}$ and half-integer $n_{g}$.

\section{B. Superconducting atomic limit}

We now turn to the atomic limit, where $\Delta$ represents the largest relevant energy scale and we can effectively put $\Delta \rightarrow \infty$. This allows us to go beyond the perturbative cotunneling regime and to compute the free energy $F$ without further approximations. Using $f(\tau) \rightarrow \Delta^{-1} \delta(\tau)$ in Eq. (2.13), the partition function reads $Z=\operatorname{Tr}_{d} e^{-\beta H_{\text {eff }}}$. The 'effective dot Hamiltonian' is

$$
H_{\mathrm{eff}}=H_{d}+\frac{1}{2} \sum_{j=L, R} \sum_{n m}\left(\Gamma_{n m}^{(j)} e^{i \phi_{j}} d_{n \downarrow} d_{m \uparrow}+\text { H.c. }\right),
$$

with $H_{d}$ in Eq. (2.1) and a proximity-induced $s$-wave pairing term due to the traced-out superconducting leads $\stackrel{3}{3}$ The CPR then follows from Eq. (2.16). Notice that the Hilbert space of the dot can now be decomposed into two independent sectors with even and odd fermion parity, respectively.

Equation (3.18) can be used to demonstrate that already in the cotunneling regime the limits $E_{c} \rightarrow \infty$ and $\Delta \rightarrow \infty$ do not commute. For $\Delta \rightarrow \infty$, one needs to retain only those contributions in Eq. (3.6) where two fermions forming a Cooper pair are tunneling as a whole, with the correlator of the form $\left\langle\mathcal{T} c_{\nu}\left(\tau+0^{+}\right) c_{\mu}(\tau) \bar{c}_{\nu}\left(\tau^{\prime}+\right.\right.$ $\left.\left.0^{+}\right) \bar{c}_{\mu}\left(\tau^{\prime}\right)\right\rangle$. Using $\tilde{E}_{\nu \mu} \geq 0$ in Eq. (3.13), some algebra gives

$$
Q_{\nu \mu}=\frac{\delta_{n_{\nu}, n_{\mu}}}{2 \Delta^{2}} \frac{1-\delta_{\tilde{E}_{\nu \mu}, 0}}{\tilde{E}_{\nu \mu}} .
$$


Since now $Q_{\nu \mu} \geq 0$ for arbitrary $N_{0}, \pi$-junction behavior is never possible in the atomic limit, in contrast to what we found for $\Delta<E_{c} \rightarrow \infty$ above. This statement always applies within the atomic limit, i.e., also beyond the cotunneling regime. Moreover, in the atomic limit, $E_{c}<\Delta \rightarrow \infty$, current flows only in the vicinity of the $2 e$-charge degenerate points, where $W_{ \pm 2}=0$ in Eq. (2.5), corresponding to integer values of $n_{g}$. This again differs from the strong-blockade result in Eq. 3.15, where current flows only for half-integer $n_{g}$. We thus conclude that the limits $E_{c} \rightarrow \infty$ and $\Delta \rightarrow \infty$ do not commute.

\section{ANOMALOUS JOSEPHSON CURRENT}

In this section, we address the CPR and, in particular, the anomalous supercurrent, $I_{a}=I(\varphi=0)$, for the twolevel dot in Sec. II C

\section{A. Cotunneling regime}

In the cotunneling regime, the currents $I_{0}$ and $I_{a}$ determining the Josephson CPR [Eq. [3.1)] follow from Eqs. (3.7) and (3.4), respectively. The anomalous supercurrent is expressed in terms of the $4 \times 4$ matrices $J$ and $Q$, see Eqs. (3.5) and (3.14), respectively, where a necessary condition for the anomalous Josephson effect is given by $J_{\nu \mu} \neq 0$ for at least one index pair $\nu>\mu$. In order to evaluate the $J$ matrix, we need the unitary matrix $U$ diagonalizing $h$. While $U$ is easily found, the lengthy result is not illuminating; we use it only to obtain the data shown in the figures below. Instead, we will provide analytical results in several complementary limits, where the algebra is simpler and allows for an intuitive picture.

Let us first observe that when the spin-orbit field is perpendicular to the Zeeman field $(\chi=\pi / 2), h$ is a symmetric matrix. Hence the diagonalizing matrix $U$ can always be chosen to have only real-valued entries, and for $\delta_{L, R}=0$, we obtain $J=0$ from Eq. (3.5). We conclude that for $\chi=\pi / 2$, the anomalous Josephson effect is only possible when at least one of the phase shifts $\delta_{L, R}$ is non-zero. This conclusion is in accordance with previous work $\stackrel{41}{n}$

\section{Collinear spin-orbit and Zeeman fields}

From Ref. 41, we then expect that the anomalous supercurrent is maximal for $\chi=0$, where spin-orbit and Zeeman fields point along the same direction. We thus consider $h$ in Eq. (2.17) for $\chi=0$, where the diagonalization matrix is

$$
U=e^{i \tau_{x} \sigma_{z} \theta / 2}, \quad \sin \theta=\frac{\alpha}{E_{d}}, \quad E_{d}=\sqrt{\epsilon^{2}+\alpha^{2}},
$$

and the spectrum $\left(E_{1}, \ldots, E_{4}\right)$ is given by $\mu+\left(E_{d}+\right.$ $B, E_{d}-B,-E_{d}+B,-E_{d}-B$ ). Using Eq. (3.3), the an-
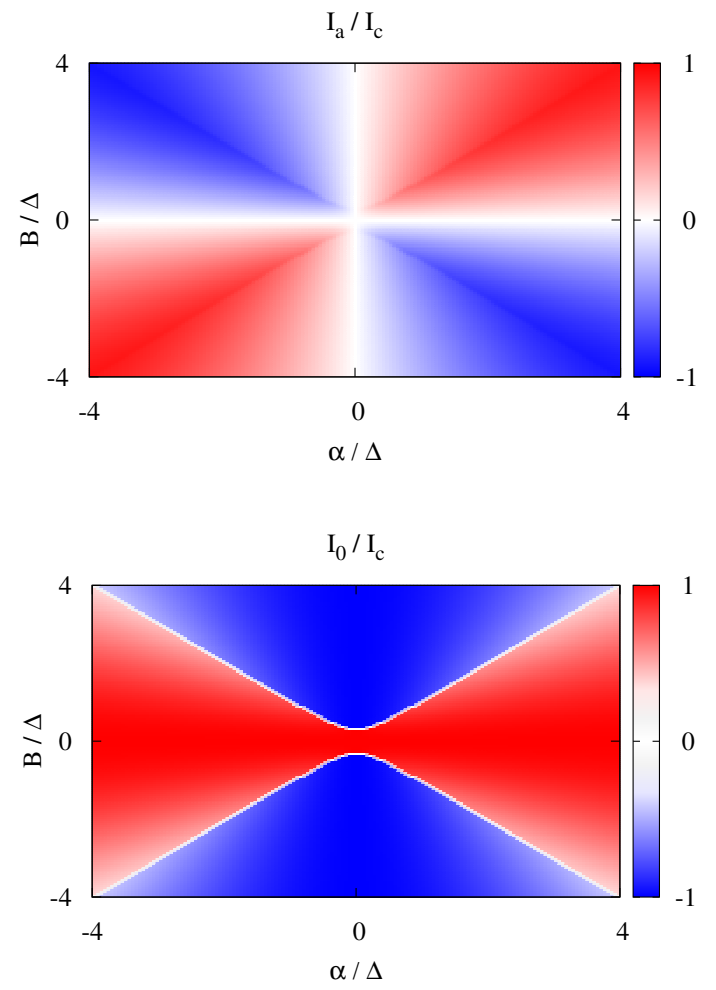

Figure 1: Anomalous supercurrent ( $I_{a}$, top panel) and 'normal' supercurrent $\left(I_{0}\right.$, bottom) determining the cotunneling CPR (3.1) in the $B-\alpha$ plane. The results are for the two-level dot with $\epsilon=0.3 \Delta, E_{c}=2 \Delta, n_{g}=2$, and $\chi=\delta_{L, R}=\mu=$ $\lambda_{L}=0$. For the right contact, only the orbital level $n=1$ is assumed to couple to the superconductor, i.e., $\lambda_{R} \rightarrow \infty$ with $\gamma_{R} e^{\lambda_{R}} \rightarrow \gamma_{R}$. Note that $I_{a, 0}$ are normalized to the respective critical current $I_{c}=\sqrt{I_{0}^{2}+I_{a}^{2}}$.

tisymmetric hybridization matrices $\tilde{\Gamma}^{(L, R)}$ have the nonvanishing entries

$\tilde{\Gamma}_{23}^{(j)}=-\left[\tilde{\Gamma}^{(j)}\right]_{14}^{*}=\gamma_{j}\left(\cos \delta_{j}+i \frac{\alpha \sinh \lambda_{j}+\epsilon \sin \delta_{j}}{E_{d}}\right)$,
$\tilde{\Gamma}_{21}^{(j)}=\left.\tilde{\Gamma}_{43}^{(j)}\right|_{\theta \rightarrow \theta+\pi}=\gamma_{j}\left(\cosh \lambda_{j}+\frac{\epsilon \sinh \lambda_{j}-\alpha \sin \delta_{j}}{E_{d}}\right)$.

The symmetric $J$ matrix in Eq. (3.5) thus has the nonzero elements

$J_{32}=\frac{\gamma_{L} \gamma_{R}}{E_{d}}\left[\epsilon \sin \delta+\alpha\left(\cos \delta_{R} \sinh \lambda_{L}-\cos \delta_{L} \sinh \lambda_{R}\right)\right]$

and $J_{41}=-J_{32}$. Remarkably, this result does not depend on the Zeeman field $B$. In the end, the anomalous supercurrent is

$$
I_{a}=\Delta^{2} J_{32}\left(Q_{32}-Q_{41}\right) .
$$

Several observations can be drawn from the above equations.

First, note that $J_{32}=0$ for $\Gamma^{(L)}=\Gamma^{(R)}$ (where $\delta_{L}=\delta_{R}$ and $\left.\lambda_{L}=\lambda_{R}\right)$. Therefore, asymmetric tunnel contacts with matrices $\Gamma^{(L)} \neq \Gamma^{(R)}$ are necessary for 

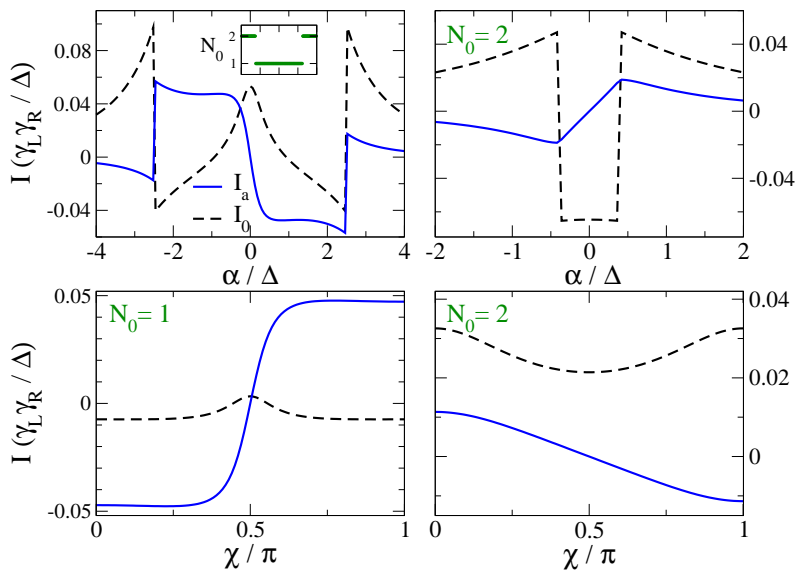

Figure 2: Parameter dependence of $I_{a, 0}$ (main panels) and of the particle number $N_{0}$ (inset) for $B=0.5 \Delta$, with other parameters as in Fig. 1 Blue solid curves show $I_{a}$, and black dashed curves $I_{0}$, both in units of $e \gamma_{L} \gamma_{R} / \hbar \Delta$. Top row: SOC $\alpha$ is varied for fixed field angle $\chi=0$, with $n_{g}=1$ (left) and $n_{g}=2$ (right). Bottom row: $\chi$ is varied for fixed $\alpha=1.2 \Delta$, with $n_{g}=1$ (left) and $n_{g}=2$ (right).
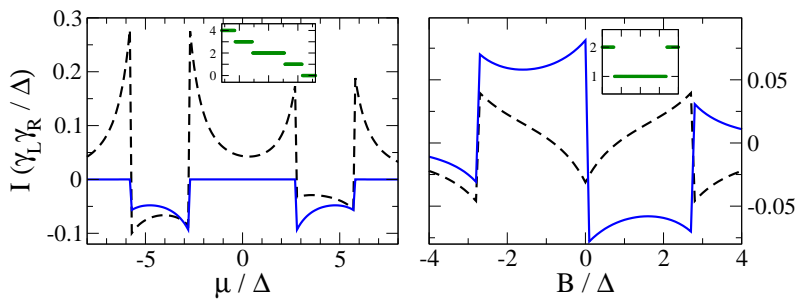

Figure 3: Same as Fig. 2 but showing $I_{a, 0}$ vs $\mu$ for $B=0.001 \Delta$ (left), and $I_{a, 0}$ vs $B$ for $\mu=3 \Delta$ (right). Other parameters are as in Fig. 1 except for $E_{c}=1.5 \Delta$.

$I_{a} \neq 0$, see Ref. 41. The resulting typical 'phase diagram' for $I_{a, 0}$ in the $B$ - $\alpha$ plane is depicted in Fig. 1 The standard Josephson effect, where one has either 0- or $\pi$ junction behavior with $\left|I_{a} / I_{0}\right| \ll 1$, is recovered when either $\alpha$ or $B$ are small. In contrast, the anomalous supercurrent is most pronounced when $|\alpha| \approx|B|$. The lower panel (for $I_{0}$ ) indicates that within the Zeemandominated regime $|B|>\sqrt{\alpha^{2}+\epsilon^{2}}$, we have $I_{0}<0$, implying that $\pi$-junction behavior can be realized. Furthermore, we observe that for the chosen parameter set, $I_{a}$ is odd in the product $\alpha B$.

The $\alpha$-dependence for fixed $B=0.5 \Delta$ is shown in the upper panel of Fig. 22 The steps in $I_{0, a}$ vs $\alpha$ (and in all figures below) can be traced back to level degeneracies, where higher-order perturbative terms become important and will smear out the steps. For the chosen parameters and $n_{g}=2$, we have $N_{0}=2$ for all shown SOCs, but for $n_{g}=1$ (upper left panel), $N_{0}=1$ for certain $\alpha$. The anomalous supercurrent is generally enhanced for odd $N_{0}$ compared to the even- $N_{0}$ case.

The lower-row panels in Fig. 2 show the $\chi$-dependence of $I_{a, 0}$ for SOC $\alpha=1.2 \Delta$, confirming that the anomalous
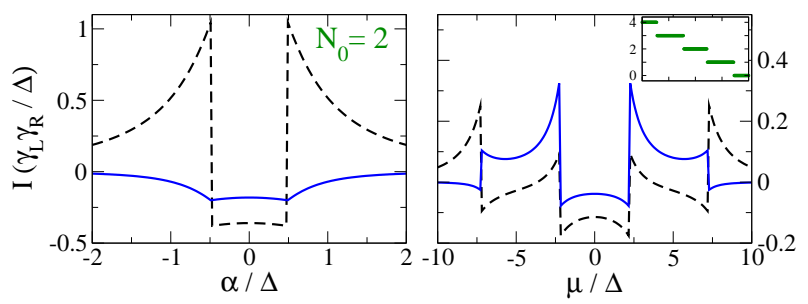

Figure 4: Same as Fig. 2 but showing $I_{a, 0}$ vs $\alpha$ for $\mu=E_{c}=0$ (left), and $I_{a, 0}$ vs $\mu$ for $\alpha=0$ and $E_{c}=2 \Delta$ (right). We use the parameters $\epsilon=0.5 \Delta, B=0.7 \Delta, n_{g}=2, \chi=\lambda_{L, R}=\delta_{R}=0$, and $\delta_{L}=\pi / 2$.

supercurrent is maximized for $\chi=0 \bmod \pi$ but vanishes for $\chi=\pi / 2$. In addition, by comparing to the respective $E_{c}=0$ plots (not shown), we observe that $I_{a}$ is not drastically affected by interactions while $I_{0}$ becomes suppressed. This suggests that interactions tend to enhance the relative importance of the anomalous supercurrent.

Next we observe that in general $Q_{32} \neq Q_{41}$. As long as $J_{32} \neq 0$, an anomalous supercurrent may then flow. This could happen for arbitrary (including zero) SOC $\alpha$. However, we always need a finite Zeeman field. Indeed, for $B=0$, we find that $Q_{32}=Q_{41}$ due to level degeneracies $\left(E_{1}=E_{2}\right.$ and $\left.E_{3}=E_{4}\right)$, and hence $I_{a}=0$ for $B=0$, cf. also Fig. 1. Nonetheless, anomalous supercurrents can survive even for arbitrarily weak $B$, in particular when interactions are present. We will address this issue in more detail below for the resonant case $(\epsilon=0)$, but Fig. 3 already illustrates the phenomenon for $\epsilon=0.3 \Delta$. The left panel in Fig. 3 shows that even for $B=0.001 \Delta$, in the presence of interactions and with odd $N_{0}$, the anomalous supercurrent is finite and sizeable. Similarly, the right panel shows that for $B \rightarrow 0$, we obtain an unusual $I_{a}(B)$ dependence instead of the standard linear $B$-dependence discussed in Ref. 41. We expect that higher-order perturbative corrections smear out the cusps near $B=0$, see also Sec. IVB, and eventually lead to $I_{a} \propto \operatorname{sgn}(B)$.

Let us now analyze the case without SOC: Putting $\alpha=0$ in Eq. (4.2), we observe that $I_{a} \neq 0$ is possible for relative inter-orbital phase shift $\delta \neq 0$, cf. Eq. (2.19). The possibility of an anomalous Josephson effect induced by the magnetic field alone (without SOC) in a noninteracting multi-level dot was overlooked in Ref. 41, where only the case $\delta_{L, R}=0$ has been studied. This effect is shown in Fig. 4 for phase shifts $\delta_{R}=0$ and $\delta_{L}=\pi / 2$ (otherwise the tunnel contacts are here assumed identical, $\lambda_{L}=\lambda_{R}$ ). The left panel, where $N_{0}=2$ for the chosen parameters, illustrates the counter-intuitive increase in $\left|I_{a}\right|$ as the SOC is decreased. In fact, here we find the largest possible anomalous supercurrent for $\alpha=0$. Note that, as a consequence of the inter-orbital phase shift $\delta=\pi / 2$, the anomalous supercurrent is now an even function of the SOC parameter $\alpha$. The right panel presents the $\mu$-dependence of $I_{a}$, where we see again that the anomalous supercurrent is enhanced whenever $N_{0}$ is odd. 
Finally, let us note that for $\delta=0$, the condition $J_{32} \neq 0$, with Eq. (4.2) for $J_{32}$, is equivalent to $\alpha \neq 0$ and nonvanishing commutator $\left[\Gamma^{(L)}, \Gamma^{(R)}\right] \neq 0$, which corresponds to the chirality condition in Sec. I. These two necessary conditions for anomalous supercurrents were specified in Ref. 41 .

\section{Resonant level}

Another interesting and nontrivial situation emerges when the two bare levels are resonantly aligned. Then $\epsilon=0$ (with arbitrary $\chi$ ) in Eq. (2.17), and the unitary matrix $U$ diagonalizing $h$ is

$$
U=e^{i \tau_{x} \pi / 4} e^{i \hat{\theta} \sigma_{x} / 2}
$$

where $\hat{\theta}=\operatorname{diag}\left(\theta_{+}, \theta_{-}\right)$is a diagonal matrix in orbital space. The angles $\theta_{ \pm}$follow from

$$
e^{i \theta_{ \pm}}=\frac{B \pm e^{i \chi} \alpha}{E_{ \pm}}, \quad E_{ \pm}=\sqrt{\alpha^{2}+B^{2} \pm 2 \alpha B \cos \chi}
$$

and $\left(E_{1}, \ldots, E_{4}\right)=\mu+\left(E_{+},-E_{+}, E_{-},-E_{-}\right)$. Some algebra shows that the symmetric $J$ matrix has the nonvanishing elements $\frac{71}{1}$

$$
J_{21}=\gamma_{L} \gamma_{R}\left(\cos \delta_{L} \sinh \lambda_{R}-\cos \delta_{R} \sinh \lambda_{L}\right)
$$

and $J_{43}=-J_{21}$. For the anomalous Josephson current, we thus find

$$
I_{a}=\Delta^{2} J_{21}\left(Q_{21}-Q_{43}\right) .
$$

Quite remarkably, $J_{21}$ in Eq. (4.6) neither depends on the Zeeman field $B$ nor on the SOC $\alpha$. In principle, we may then expect $I_{a} \neq 0$ even for very small $\alpha$ and/or $B$. In addition, $J_{21}$ does not depend on $\chi$ either, and it is not obvious why $I_{a}=0$ for $\chi=\pi / 2$ as discussed above. However, we also need to examine the contribution of the $Q$ matrix. In fact, when $\alpha B \cos \chi=0$, the level degeneracy $E_{+}=E_{-}$implies from Eq. (3.14) that $Q_{21}=$ $Q_{43}$, which in turn gives $I_{a}=0$ for $\epsilon=0$ and arbitrary $E_{c}$.

Nonetheless, we again encounter the possibility that $I_{a} \neq 0$ even for very small Zeeman field $B$ and temperatures $T<|B|$, suggesting the incipient spontaneous breakdown of TRS (note that TRS is restored for $B=0$ ). Remarkably, this onset behavior can be triggered by Coulomb interactions even for very small SOC $\alpha$. Before going through the detailed argument, we first illustrate this behavior for $B=0.001 \Delta$ in Fig. [5 The left panel indeed reveals a finite and sizeable anomalous supercurrent for $\alpha=B=0.001 \Delta$ if interactions are present, $E_{c} \neq 0$, and $N_{0}$ is odd. The right panel suggests that $I_{a} \propto \operatorname{sgn}(\alpha B)$ for arbitrarily small (but finite) $\alpha$. For $\alpha=0.5 \Delta$, the interaction effects in this interesting parameter regime are displayed in Fig. 6. While $I_{a}=0$ for small $E_{c}$, we find $I_{a} \neq 0$ for $E_{c} \gtrsim|\alpha|$, with $\left|I_{a}\right|$ weakly
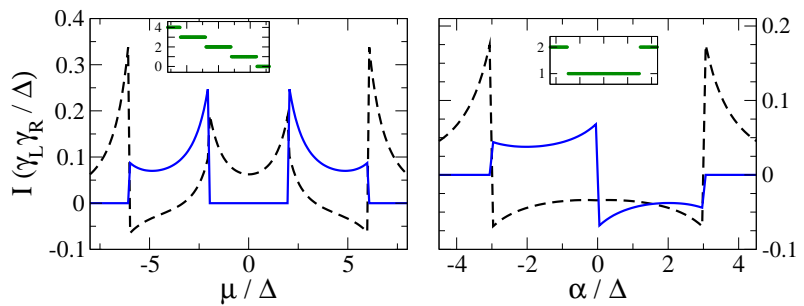

Figure 5: Same as Fig. 2 but for the resonant orbital $(\epsilon=0)$ case with tiny Zeeman field, $B=0.001 \Delta$. The left panel shows $I_{a, 0}$ vs $\mu$ for $\alpha=0.001 \Delta$, while the right panel displays $I_{a, 0}$ vs $\alpha$ for $\mu=5 \Delta$. The remaining parameters are as in Fig. 1.

decreasing in the limit of strong Coulomb blockade. For the resonant case of half-integer $n_{g}, I_{a}$ saturates at a finite value for $E_{c} \rightarrow \infty$, cf. inset of Fig. [6.

Next we aim at understanding the above $I_{a} \propto \operatorname{sgn}(\alpha B)$ onset behavior. To simplify the algebra as much as possible, we put $\chi=0$ and consider the limiting case of very small but finite $(B, \alpha)$, where interactions play a crucial role. (For $|\alpha| \gg|B|$, the arguments below show that the onset behavior $I_{a} \propto \operatorname{sgn}(B)$ is possible even when $E_{c}=0$.) Equation (4.5) then gives $e^{i \theta_{ \pm}}= \pm \operatorname{sgn}(\alpha)$ for $|\alpha|>|B|$, and thus the complex-valued unitary matrix in Eq. (4.4) has different limits for positive and negative SOC, $\lim _{\alpha \rightarrow 0^{+}} U \neq \lim _{\alpha \rightarrow-0^{+}} U$. This corresponds to different residual 'magnetizations' of the $\tau \otimes \sigma$ isospin near the $S U(4)$ symmetric point in parameter space defined by $B=\alpha=0$. (Note that in the absence of hysteresis, $I_{a}=0$ directly at the symmetric point, since then $U=\operatorname{diag}(1)$ is real-valued and thus implies $J=0$.) Recall next that the columns of $U$ are eigenvectors of $h$, forming four linearly independent isospin projections. The corresponding single-particle energy levels are $\mu+\{|\alpha|+\eta,-|\alpha|-\eta,|\alpha|-\eta,-|\alpha|+\eta\}$ with $\eta=\operatorname{sgn}(\alpha) B$. When $\mu$ is chosen such that $N_{0}=1$, assuming $B>0$, one spin- $\downarrow$ electron will occupy the singleparticle level $E_{2}\left(E_{4}\right)$ for $\alpha>0(\alpha<0)$. For $N_{0}=1$, we observe that $\operatorname{sgn}\left(Q_{21}\right)=-\operatorname{sgn}\left(Q_{43}\right)=-\operatorname{sgn}(\alpha)$, see Eq. (3.14) with $\mathcal{Q}_{i}>0$, and therefore Eq. (4.7) suggests that we may have a finite anomalous supercurrent. However, for very small $(B, \alpha)$ and $E_{c}=0$, the energy separation between states with different $N_{0}$ is also tiny. This eventually results in the complete cancellation of all time-reversed contributions, and $I_{a}=0$ in the noninteracting case for very small $B$ and $\alpha$. For finite charging energy, however, the energy gap to states with different $N_{0}$ grows with $E_{c}$, which renders the $N_{0}=1$ ground state more robust. Taking the small- $(B, \alpha)$ limit for finite $E_{c}$ should then leave ground-state properties such as $N_{0}$ or the spin polarization unaffected, and $I_{a} \propto \operatorname{sgn}(\alpha B)$ remains finite. However, the above arguments also show that $I_{a}$ will be suppressed by thermal fluctuations once the temperature scale exceeds the Zeeman field scale. Therefore the $I_{a} \propto \operatorname{sgn}(\alpha B)$ onset behavior just found for the ground state can 'only' be interpreted as incipi- 


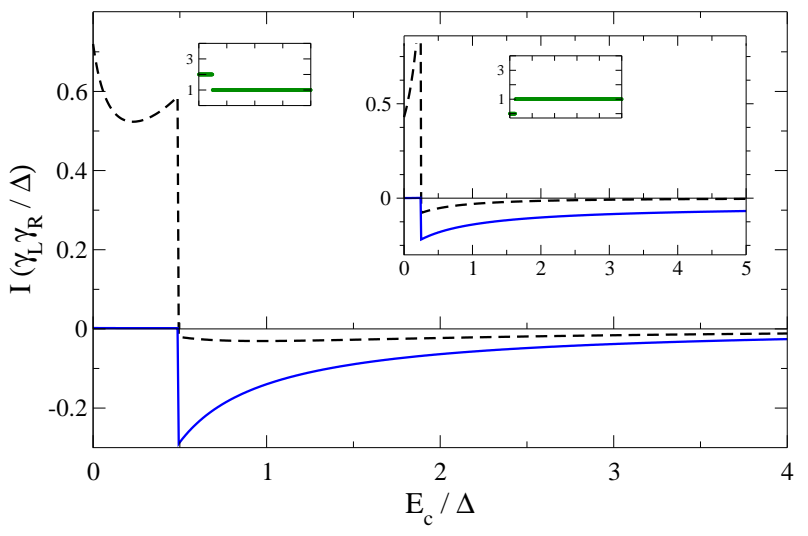

Figure 6: Same as Fig. 2 but showing $I_{a, 0}$ vs $E_{c}$ for $n_{g}=2$ (main panel) and $n_{g}=3 / 2$ (large right inset), with $\alpha=0.5 \Delta$, $B=0.01 \Delta$, and $\epsilon=0.01 \Delta$.

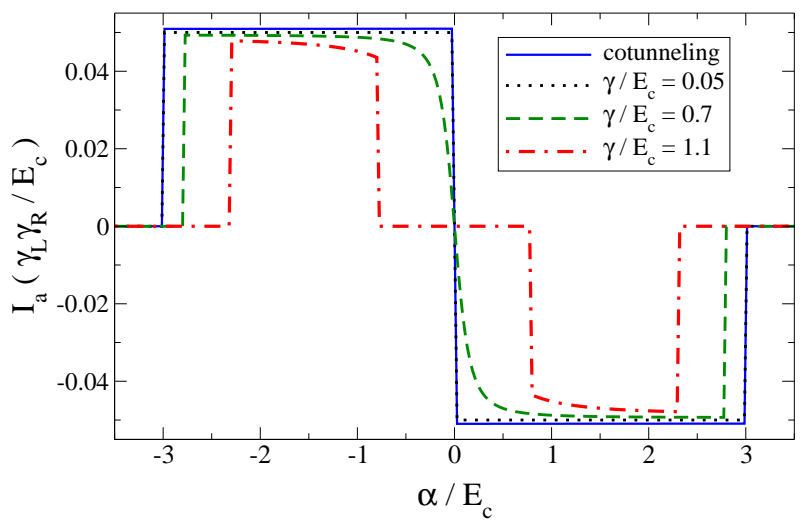

Figure 7: Anomalous supercurrent $I_{a}$ for the two-orbital dot vs SOC $\alpha$ in the atomic limit $(\Delta \rightarrow \infty)$ for several $\gamma=\gamma_{L}=$ $\gamma_{R}$. The shown results follow from Eq. 4.9) and the effective dot Hamiltonian (3.18). The other parameters are as in the right panel of Fig. [5] $\epsilon=0, B / E_{c}=0.0005, \mu / E_{c}=2.5$, $n_{g}=2, \chi=\delta_{L, R}=\lambda_{L}=0$, and $\lambda_{R} \rightarrow \infty$. The solid blue curve gives the respective cotunneling result [Eq. (4.7] with $\Delta \rightarrow \infty]$ for $\gamma / E_{c}=0.05$.

ent breakdown of TRS, i.e., TRS is restored by thermal fluctuations for $T>|B|$.

Analytical results for the ground-state anomalous supercurrent are possible in the strong Coulomb blockade limit. For instance, at the charge degeneracy point $n_{g}=3 / 2$ with $N_{0}=1$, Eq. (4.7) yields for small $(B, \alpha)$ the result

$$
I_{a}=-3 \operatorname{sgn}(\alpha B) \Delta^{2} J_{21} \mathcal{Q}_{3}(\mu, 0, \mu),
$$

where $J_{21}$ and $\mathcal{Q}_{3}(\mu, 0, \mu)$ are given in Eqs. (4.6) and (3.17), respectively. This confirms explicitly the $I_{a} \propto$ $\operatorname{sgn}(\alpha B)$ onset behavior discussed above.

\section{B. Superconducting atomic limit}

Next we briefly turn to a discussion of the anomalous Josephson effect in the superconducting atomic limit, see Sec. IIIB where the $\Delta \rightarrow \infty$ effective dot Hamiltonian, $H_{\text {eff }}$ in Eq. (3.18), allows us to go beyond the perturbative cotunneling regime. Evaluating the anomalous Josephson current at the, say, left contact, we obtain

$$
I_{a}=-\frac{2 e}{\hbar} \operatorname{Im} \sum_{\nu<\mu} \tilde{\Gamma}_{\nu \mu}^{(L)}\left\langle c_{\nu} c_{\mu}\right\rangle,
$$

where the brackets indicate a ground-state average using $H_{\text {eff }}(\varphi=0)$. We consider the two-orbital dot in Sec. IIC, where the $4 \times 4$ hybridization matrices $\tilde{\Gamma}^{(L, R)}$ follow from Eq. (2.18) after transformation to the $c_{\nu}$ fermion representation. As detailed in Sec. IIIB the $\varphi$-dependent ground-state energies should be computed separately for the (decoupled) odd and even fermion parity subspaces. We then expect $I_{a} \neq 0$ only when the ground state (for $\varphi=0$ ) has odd parity.

The dependence of $I_{a}$ on the SOC $\alpha$ is illustrated in Fig. 7, where we use parameters as in the right panel of Fig. 5. This allows us to study how the $I_{a} \propto \operatorname{sgn}(\alpha B)$ onset behavior (the signature of incipient TRS breaking) emerges from the cusp features encountered in perturbation theory. First, we note from Fig. 7 that the cotunneling result (taking $\Delta \rightarrow \infty$ in the above expressions) matches the predictions of Eq. (4.9) for $\gamma_{L, R} \rightarrow 0$. This matching has also been confirmed analytically by perturbative expansion of the general $\Delta \rightarrow \infty$ cotunneling result [see Eqs. (3.4) and (3.19)] to lowest nontrivial order in the hybridization matrices. We conclude that the limits $\gamma_{L, R} \rightarrow 0$ and $\Delta \rightarrow \infty$ commute. Second, cusplike features as seen in the right panel of Fig. 5 emerging under a perturbative theory will be smeared out by higher-order corrections, and indeed imply $I_{a} \propto \operatorname{sgn}(\alpha B)$ onset behaviors associated with time-reversal symmetry breaking. Third, for large hybridizations $\gamma_{L, R}$, it is also possible that the fermion parity of the resulting $\varphi=0$ ground state is changed. This is apparent in Fig. [7 where we find $I_{a}=0$ for small $|\alpha|$ and $\gamma / E_{c}=1.1$ as a consequence of such a transition. The anomalous supercurrent can here be tuned to zero either by raising $\gamma$ or by lowering $E_{c}$.

\section{MAJORANA FERMIONS}

We proceed by noting that all ingredients needed for the realization of Majorana fermions ${ }^{24.25}$ are in principle present in our model, namely proximity-induced superconductivity, SOC, and a TRS-breaking magnetic field. As discussed below, the Majorana regime can be reached in the superconducting atomic limit of the two-level dot in Sec. IIC where the two orbitals here correspond to two spatially separated single-level dots (i.e., a double dot). The resulting MBSs are topologically unprotected, i.e., 
their realization requires the fine-tuning of gate voltages, Zeeman field $B$, and/or phase difference $\varphi$. Gate voltages here affect the orbital asymmetry $\epsilon$ through confinement potentials, the average energy $\mu$, and/or the SOC $\alpha$. For a spatially separated MBS pair - such that both MBSs correspond to different orbital states, allowing to distinguish them - we find characteristic signatures in the $2 \pi$-periodic CPR. This is in marked contrast to the 'fractional' $4 \pi$-periodic CPR for topologically protected Majoranas, 25 which has not been observed so far due to difficulties in ensuring fermion parity conservation in practice.

We use the atomic-limit effective Hamiltonian $H_{\text {eff }}$ in Eq. (3.18) for the double dot. Using the basis $\{|1, \uparrow\rangle, \mid 2, \downarrow$ \rangle$,|1, \downarrow\rangle,|2, \uparrow\rangle\}$, the single-particle matrix $h$ [Eq. (2.17] has the representation

$h=\left(\begin{array}{cccc}\mu+\epsilon+B & -\alpha \sin \chi & 0 & i \alpha \cos \chi \\ -\alpha \sin \chi & \mu-(\epsilon+B) & i \alpha \cos \chi & 0 \\ 0 & -i \alpha \cos \chi & \mu+\epsilon-B & \alpha \sin \chi \\ -i \alpha \cos \chi & 0 & \alpha \sin \chi & \mu-(\epsilon-B)\end{array}\right)$.

Without losing generality, $\alpha>0$ and $B>0$ from now on. We approach a suitable parameter regime by comparing to the Kitaev chain ${ }^{23}-26$ describing 1D (effectively spinless) $p$-wave topological superconductors. The Kitaev chain is known to support MBSs, and based on this analogy we choose $\chi=\pi / 2$, i.e., Zeeman and spin-orbit fields are perpendicular $\frac{25}{2} h$ is then block-diagonal with decoupled upper and lower two-state subspaces. The connection to the Kitaev chain becomes clear when $\epsilon$ is positive and chosen in the parameter regime

$$
\Delta \gg \epsilon+B \gg \max \left(\alpha,|\epsilon-B|, \gamma_{L, R}, \mu, E_{c}\right) .
$$

The upper-block state $(2, \downarrow)$ will then always be occupied, while $(1, \uparrow)$ is always empty. The upper left block in Eq. (5.1) can thus be projected away, and the resulting truncated Hamiltonian, $H_{\text {eff }}^{\prime}$, acts only within the lower right block described by the (effectively spinless) fermion operators $d_{1} \equiv d_{1 \downarrow}$ and $d_{2} \equiv d_{2 \uparrow}$,

$$
\begin{aligned}
H_{\mathrm{eff}}^{\prime} & =(\mu+\epsilon-B) d_{1}^{\dagger} d_{1}+[\mu-(\epsilon-B)] d_{2}^{\dagger} d_{2} \\
& +E_{c}\left(d_{1}^{\dagger} d_{1}+d_{2}^{\dagger} d_{2}-n_{g}\right)^{2} \\
& +\left(\alpha d_{1}^{\dagger} d_{2}+\tilde{\Delta}(\varphi) e^{i \vartheta(\varphi)} d_{2}^{\dagger} d_{1}^{\dagger}+\text { H.c. }\right),
\end{aligned}
$$

where the occupied $(2, \downarrow)$ state leads to a shift $n_{g} \rightarrow n_{g}+$ 1. With the hybridization matrix (2.18), the double dot model in Eq. (3.18) yields the complex-valued effective pairing amplitude $\widetilde{\Delta} e^{i \vartheta}=\frac{1}{2} \sum_{j} \gamma_{j} e^{-i\left(\phi_{j}+\delta_{j}\right)}$. It is now convenient to introduce $\gamma \equiv\left(\gamma_{L}+\gamma_{R}\right) / 2$, and to gauge away the overall phase $\sum_{j}\left(\phi_{j}+\delta_{j}\right) / 2$. We then obtain

$$
\begin{gathered}
\tilde{\Delta}(\varphi)=\gamma \sqrt{1-T_{0} \sin ^{2}[(\varphi+\delta) / 2]}, \\
T_{0}=\frac{4 \gamma_{L} \gamma_{R}}{\left(\gamma_{L}+\gamma_{R}\right)^{2}}, \\
\vartheta(\varphi)=\tan ^{-1}\left(\frac{\gamma_{R}-\gamma_{L}}{\gamma_{R}+\gamma_{L}} \tan [(\varphi+\delta) / 2]\right),
\end{gathered}
$$

with the phase shift $\delta$ in Eq. (2.19). Note that $0 \leq T_{0} \leq 1$ corresponds to the transmission probability of a singlechannel quantum point contact, while $\tilde{\Delta}(\varphi)$ gives the Andreev level energy in the atomic limit 65

We proceed by first discussing the noninteracting case, $E_{c}=0$, where two spatially resolved MBSs may appear when the (necessary) conditions

$$
B=\epsilon, \quad \mu=0
$$

are met. $H_{\mathrm{eff}}^{\prime}$ can then be diagonalized in terms of fermionic Bogoliubov-de Gennes (BdG) quasiparticle operators,

$$
\eta_{ \pm}=\frac{1}{2}\left[d_{1}+d_{2} \pm e^{i \vartheta}\left(d_{1}^{\dagger}-d_{2}^{\dagger}\right)\right]
$$

where Eq. (5.3) yields the BdG Hamiltonian

$$
H_{\mathrm{eff}}^{\prime}=\sum_{ \pm} E_{ \pm}(\varphi)\left(\eta_{ \pm}^{\dagger} \eta_{ \pm}-\frac{1}{2}\right), \quad E_{ \pm}=\alpha \pm \tilde{\Delta}(\varphi)
$$

The four possible single-particle eigenstates are constructed by applying $\eta_{ \pm}^{\dagger}$ or $\eta_{ \pm}$to the vacuum state, with the respective energies $E_{ \pm} / 2$ and $-E_{ \pm} / 2$. The CPR then follows from Eq. (5.7),

$$
I(\varphi)=2 \frac{\partial \tilde{\Delta}}{\partial \varphi}\left[\Theta\left(-E_{+}\right)-\Theta\left(-E_{-}\right)\right]
$$

where $\Theta$ is the Heaviside function. Notice that $I=0$ for $\tilde{\Delta}(\varphi)<\alpha$, since both energies $E_{ \pm}=\alpha \pm \tilde{\Delta}$ have the same sign. We therefore find

$$
\begin{aligned}
I(\varphi) & =\Theta(\tilde{\Delta}-\alpha) I_{0}(\varphi) \\
I_{0}(\varphi) & =\frac{e \gamma}{2 \hbar} \frac{T_{0} \sin (\varphi+\delta)}{\sqrt{1-T_{0} \sin ^{2}[(\varphi+\delta) / 2]}}
\end{aligned}
$$

where $I_{0}(\varphi)$ coincides with the CPR of a single-channel quantum point contact with transparency $T_{0}, \frac{3}{,}$ shifted by the inter-orbital phase difference $\delta$. The CPR (5.9) is $2 \pi$ periodic in $\varphi$ and vanishes (or reappears) at the boundaries between ground states with opposite fermion parity. These boundaries are precisely the formation points of MBSs, as we show next.

Noting that both $\alpha$ and $\tilde{\Delta}$ are non-negative, the zero-energy condition for MBS formation is satisfied for $E_{-}(\varphi)=0$, i.e., for

$$
\tilde{\Delta}(\varphi)=\alpha
$$

This corresponds to a pair of zero-energy MBSs, generated by the anticommuting Majorana fermion operators $\xi_{1}=-i\left(\eta_{-}-\eta_{-}^{\dagger}\right)$ and $\xi_{2}=\eta_{-}+\eta_{-}^{\dagger}$; note that $\xi_{n}=\xi_{n}^{\dagger}$ and $\xi_{n}^{2}=1$. In order to avoid recombination to a conventional fermion, we need both MBSs to be spatially separated. This is achieved for

$$
\vartheta(\varphi)=0 \bmod \pi
$$


where $\xi_{1}$ and $\xi_{2}$ have well-defined and different orbital quantum numbers, and thus correspond to different single-level dots in this double dot. Taking for instance $\vartheta=0$, Eq. (5.6) yields $\xi_{1}=-i\left(d_{1}-d_{1}^{\dagger}\right)$ and $\xi_{2}=d_{2}+d_{2}^{\dagger}$, which indeed implies that the MBS associated with $\xi_{n=1(2)}$ has the orbital wavefunction $n=1$ (2). We conclude that Eq. (5.11) ensures that both MBSs are spatially separated. Using Eq. (5.4), there are two possibilities to satisfy this condition: (1) We may choose equal hybridization strengths, $\gamma_{L}=\gamma_{R}=\gamma$. Then $T_{0}=1$, which implies $\tilde{\Delta}=\gamma|\cos [(\varphi+\delta) / 2]|=\alpha$, with two solutions (for $\varphi$ ) when $\gamma>\alpha$. For these two phase values, MBSs will be present. (2) Alternatively, for $\gamma_{L} \neq \gamma_{R}$, another possibility emerges by adjusting $\varphi=-\delta(\bmod$ $2 \pi$ ), where Eq. (5.10) allows for a MBS pair when $\gamma=\alpha$. Realizing either of those conditions amounts to reaching the 'sweet spot' for a Kitaev chain with two fermion sites, see also Refs. 62 64. The MBS solutions are here quadratically protected against small deviations in the effective dot levels, see Eq. (5.5). While there is no such protection against deviations from the condition (5.10), this lack of protection also offers the advantage of MBS tunability by variation of the superconducting phase difference. Noting that already for a three-site chain, robust protection of unpaired MBSs can be achieved, $\stackrel{63}{=}$ we expect that a reasonable compromise between well protected MBSs and good tunability is possible using our double dot proposal.

Let us now see how the above scenario will be affected by weak electron-electron interactions. We here continue to use the 'global charging energy' in Eq. (2.1), since for a double dot in the large- $B$ limit of interest here, see Eq. (5.2), both dots are effectively occupied by one fermion at most. In that case, the global charging energy is equivalent to a capacitive inter-dot interaction. For finite $E_{c}$, the system can be tuned to the MBS regime by replacing the condition $\mu=0$ in Eq. (5.5) by $\mu=$ $-2 E_{c}\left(1-n_{g}\right)$, i.e., by putting $\mu$ at the charge degeneracy point. ( $B=\epsilon$ is still required.) In terms of the $\eta_{ \pm}$ operators in Eq. (5.6), the Hamiltonian (5.3) then reads

$$
H=\sum_{ \pm} E_{ \pm}(\varphi)\left(\eta_{ \pm}^{\dagger} \eta_{ \pm}-\frac{1}{2}\right)+E_{c}\left(\eta_{+}^{\dagger} \eta_{+}-\eta_{-}^{\dagger} \eta_{-}\right)^{2}
$$

with $E_{ \pm}(\varphi)$ in Eq. (5.7). The MBS regime is realized when there are two ground states with opposite fermion parity. By examining the many-particle spectrum of Eq. (5.12),

$$
\begin{aligned}
& E_{0,0}=-\alpha, \quad E_{1,0}=\tilde{\Delta}+E_{c} \\
& E_{0,1}=-\tilde{\Delta}+E_{c}, \quad E_{1,1}=\alpha,
\end{aligned}
$$

where $E_{n_{+}, n_{-}}$denotes the energy of a state with $n_{ \pm}=$ $\left\langle\eta_{ \pm}^{\dagger} \eta_{ \pm}\right\rangle$, the condition (5.10) for the appearance of MBSs is replaced by

$$
\alpha=\tilde{\Delta}(\varphi)-E_{c}>0 .
$$

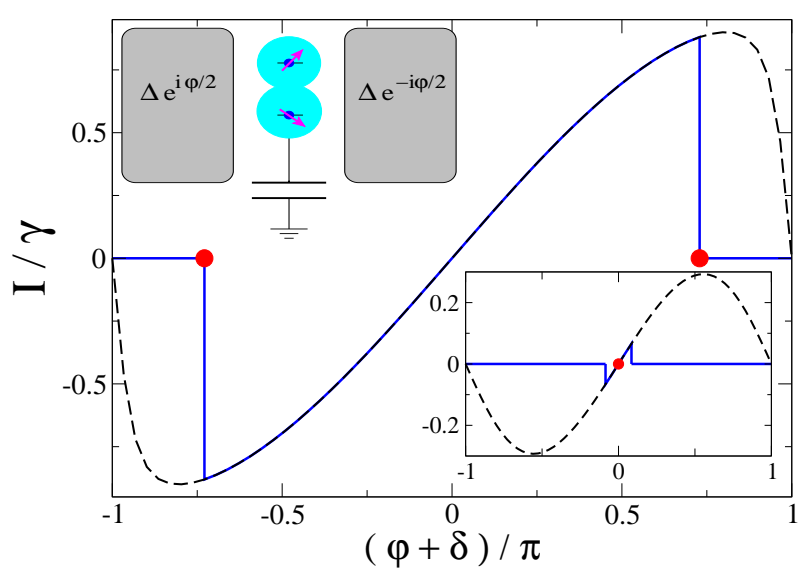

Figure 8: CPR through a double dot in the atomic limit [see Eq. [5.2)] with $B=\epsilon, \mu=E_{c}=0$, and $\chi=\pi / 2$. Main panel: CPR (blue solid curve) for $\alpha=0.4 \gamma$, where $\gamma=\left(\gamma_{L}+\right.$ $\left.\gamma_{R}\right) / 2$ with slightly asymmetric $\gamma_{R, L}$ such that $T_{0}=0.99$. Red points on the CPR indicate that for the respective value of $\varphi$, a MBS pair is formed (see main text). The dashed black curve shows the CPR for $\alpha=0$, where no MBSs occur. The top left inset shows the schematic setup. The bottom right inset gives the CPR for $\alpha=0.99 \gamma$ and significant hybridization asymmetry, $T_{0}=0.5$, as blue solid curve. The red point indicates MBS pair formation, and the dashed curve is for $\alpha=0$ (without MBSs).

In the MBS regime, one has a double-degenerate ground state, corresponding to negative energy eigenvalues $E_{0,1}=E_{0,0}$. Inclusion of the charging energy thus only shifts the conditions for Majorana formation, and below we focus on the case $E_{c}=0$. Our proposal is therefore rather different from the double-dot scenario in Ref. 62, where MBSs are induced only in the limit of strong intradot Coulomb interactions while the magnetic Zeeman field can be arbitrarily small.

The Josephson current [Eq. (5.9)] turns out to be nonzero (zero) for odd (even) $N_{0}$, where the CPR in general consists of two different regions: For $\tilde{\Delta}(\varphi)>\alpha$, we find $I=I_{0}(\varphi)$ as for a single-channel quantum point contact (but with a phase shift when $\delta \neq 0$ ), while $I=0$ for $\tilde{\Delta}<\alpha$. At the boundary between both regions, the parity $(-)^{N_{0}}$ changes from odd to even (or vice versa). It is precisely at these points that two degenerate 'halffermion' BdG quasi-particle states appear. Under the described conditions, these can form a pair of spatially separated MBSs. Observation of $I=0$ within a part of the CPR can then serve as indirect signature for the MBSs, as illustrated in Fig. 8. While jumps in the CPR can also have a different origin, the peculiar feature linked to the appearance of MBS pairs is the complete vanishing of the supercurrent in a finite phase interval. For the asymmetric case shown in the lower inset of Fig. 8 , the other two points on the CPR where the current vanishes correspond to spatially overlapping MBSs. 
Ideally, one should thus consider a symmetric setup with $T_{0}=1$ in order to satisfy Eq. (5.11). The MBSs can then be detected through parity changes causing abrupt current jumps in the CPR. In contrast, for asymmetric cases with $T_{0}<1$, Eq. (5.11) is satisfied only at $\varphi=-\delta$, where MBSs cannot be detected via transport measurements. With decreasing transparency $T_{0}$, corresponding to increasing overlap between both MBSs [note that $\vartheta$ in Eq. (5.4) is a function of $T_{0}$ ], the critical current decreases and the flat region $(I=0)$ in the CPR gets shorter. In fact, Eq. (5.9) predicts that for transparencies $T_{0}<T_{c}$, with a critical transparency value determined by $\gamma \sqrt{1-T_{c}^{2}}=\alpha$, there will be no flat CPR regions, and hence no abrupt current jumps, at all. Finally, it is worthwhile pointing out that in contrast to the fractional Josephson effect for topologically protected Majoranas, the MBSs discussed here do not mediate a Josephson current themselves.

\section{CONCLUSIONS}

In this work, we have analyzed two particularly interesting aspects of Josephson transport in hybrid superconductor-dot systems - a pair of conventional BCS superconductors connected through a multi-level quan- tum dot - where SOC, Coulomb charging and magnetic field effects are taken into account. First, we have studied the conditions for the anomalous Josephson effect, i.e., supercurrent flow for vanishing phase difference. It is remarkable that Coulomb interactions can qualitatively affect this phenomenon to allow for ground-state anomalous supercurrents even when time-reversal breaking perturbations are very small compared to all other relevant scales. As described in Sec. IV, we find spontaneously broken time-reversal symmetry with anomalous supercurrent flowing for arbitrarily weak Zeeman fields. Second, in the deep subgap case, we have determined the conditions for observing a pair of topologically unprotected yet spatially separated Majorana bound states in a double dot. The formation of such exotic particles is presently under vigorous study and could be indirectly detected in the CPR through the critical phases $\varphi$, where the current switches from a finite value to zero. We hope that these effects can soon be observed in experiments.

\section{Acknowledgments}

We thank Roland Hützen for help in preparing the figures. This work has been supported by the DFG (Grant No. EG 96/9-1).
1 A.A. Golubov, M.Yu. Kupriyanov, and E. Il'ichev, Rev. Mod. Phys. 76, 411 (2004).

2 S. Di Franceschi, L. Kouwenhoven, C. Schönenberger, and W. Wernsdorfer, Nature Nanotech. 5, 703 (2010).

3 A. Martín-Rodero and A. Levy Yeyati, Adv. Phys. 60, 899 (2011).

4 H. Takayanagi, T. Akazaki, and J. Nitta, Phys. Rev. Lett. 75, 3533 (1995).

5 Th. Schäpers, V.A. Guzenko, R.P. Müller, A.A. Golubov, A. Brinkman, G. Crecilius, A. Kaluza, and H. Lüth, Phys. Rev. B 67, 014522 (2003).

${ }^{6}$ M. Ebel, C. Busch, U. Merkt, M. Grajcar, T. Plecenik, and E. Il'ichev, Phys. Rev. B 71, 052506 (2005).

7 J. Xiang, A. Vidan, M. Tinkham, R.M. Westervelt, and C.M. Lieber, Nature Nanotech. 1, 208 (2006).

8 S. Tirelli, A.M. Savin, C.P. Garcia, J.P. Pekola, F. Beltram, and F. Giazotto, Phys. Rev. Lett. 101, 077004 (2008).

9 M.L. Della Rocca, M. Chauvin, B. Huard, H. Pothier, D. Esteve, and C. Urbina, Phys. Rev. Lett. 99, 127005 (2007).

10 J.D. Pillet, C.H.L. Quay, P. Morfin, C. Bena, A. Levy Yeyati, and P. Joyez, Nature Phys. 6, 965 (2010).

11 R. Winkler, Spin-Orbit Coupling Effects in TwoDimensional Electron and Hole Systems (Springer, New York, 2003).

12 Y.J. Doh, J.A. van Dam, A.L. Roest, E.P.A.M. Bakkers, L.P. Kouwenhoven, and S. De Franceschi, Science 309, 272 (2005).

13 J.A. van Dam, Yu.V. Nazarov, E.P.A.M. Bakkers, S. De Franceschi, and L.P. Kouwenhoven, Nature 442, 667 (2006).
14 T. Sand-Jespersen, J. Paaske, B.M. Andersen, K. GroveRasmussen, H.I. Jørgensen, M. Aagesen, C.B. Sørensen, P.E. Lindelof, K. Flensberg, and J. Nygård, Phys. Rev. Lett. 99, 126603 (2007).

15 R.S. Deacon, Y. Tanaka, A. Oiwa, R. Sakano, K. Yoshida, K. Shibata, K. Hirakawa, and S. Tarucha, Phys. Rev. Lett. 104, 076805 (2010).

16 Y. Kanai, R.S. Deacon, A. Oiwa, K. Yoshida, K. Shibata, K. Hirakawa, and S. Tarucha, Phys. Rev. B 82, 054512 (2010); Y. Kanai, R.S. Deacon, S. Takahashi, A. Oiwa, K. Yoshida, K. Shibata, K. Hirakawa, Y. Tokura, and S. Tarucha, Nature Nanotech. 6, 511 (2011).

17 E.J.H. Lee, X. Jiang, R. Aguado, G. Katsaros, C.M. Lieber, and S. De Franceschi, Phys. Rev. Lett. 109, 186802 (2012).

18 W. Chang, V.E. Manucharyan, T.S. Jespersen, J. Nygård, and C.M. Marcus, Phys. Rev. Lett. 110, 217005 (2013).

19 E.J.H. Lee, X. Jiang, M. Houzet, R. Aguado, C.M. Lieber, and S. De Franceschi, preprint arXiv:1302.2611

${ }^{20}$ H.A. Nilsson, P. Samuelsson, P. Caroff, and H.Q. Xu, Nano Lett. 12, 228 (2012).

21 G. Katsaros, P. Spathis, M. Stoffel, F. Fournel, M. Mongillo, V. Bouchiat, F. Lefloch, A. Rastelli, O.G. Schmidt, and S. De Franceschi, Nature Nanotech. 5, 458 (2010).

22 M.Z. Hasan and C.L. Kane, Rev. Mod. Phys. 82, 3045 (2010).

23 X.L. Qi and S.C. Zhang, Rev. Mod. Phys. 83, 1057 (2011).

24 C.W.J. Beenakker, Annu. Rev. Con. Mat. Phys. 4, 113 (2013).

25 J. Alicea, Rep. Prog. Phys. 75, 076501 (2012). 
26 M. Leijnse and K. Flensberg, Semicond. Sci. Techn. 27, 124003 (2012).

27 R.M. Lutchyn, J.D. Sau, and S. Das Sarma, Phys. Rev. Lett. 105, 077001 (2010).

28 Y. Oreg, G. Refael, and F. von Oppen, Phys. Rev. Lett. 105, 177002 (2010).

29 V. Mourik, K. Zuo, S.M. Frolov, S.R. Plissard, E.P.A.M. Bakkers, and L.P. Kouwenhoven, Science 336, 1003 (2012).

${ }^{30}$ L. Rokhinson, X. Liu, and J. Furdyna, Nature Phys. 8, 795 (2012).

31 A. Das, Y. Ronen, Y. Most, Y. Oreg, M. Heiblum, and H. Shtrikman, Nature Phys. 8, 887 (2012).

32 M.T. Deng, C.L. Yu, G.Y. Huang, M. Larsson, P. Caroff, and H.Q. Xu, Nano Lett. 12, 6414 (2012).

33 E.V. Bezuglyi, A.S. Rozhavsky, I.D. Vagner, and P. Wyder, Phys. Rev. B 66, 052508 (2002).

34 I.V. Krive, L.Y. Gorelik, R.I. Shekhter, and M. Jonson, Fiz. Nizk. Temp. 30, 535 (2004) [Low Temp. Phys. 30, 398 (2004)].

${ }^{35}$ I.V. Krive, A.M. Kadigrobov, R.I. Shekhter, and M. Jonson, Phys. Rev. B 71, 214516 (2005).

${ }^{36}$ O.V. Dimitrova and M.V. Feigel'man, JETP 102, 652 (2006).

37 L. Dell'Anna, A. Zazunov, R. Egger, and T. Martin, Phys. Rev. B 75, 085305 (2007).

38 B. Béri, J.H. Bardarson, and C.W.J. Beenakker, Phys. Rev. B 77, 045311 (2008).

39 A.A. Reynoso, G. Usaj, C.A. Balseiro, D. Feinberg, and M. Avignon, Phys. Rev. Lett. 101, 107001 (2008).

40 A. Buzdin, Phys. Rev. Lett. 101, 107005 (2008).

41 A. Zazunov, R. Egger, T. Jonckheere, and T. Martin, Phys. Rev. Lett. 103, 147004 (2009).

42 J.F. Liu and K.S. Chan, Phys. Rev. B 82, 125305 (2010).

43 A.A. Reynoso, G. Usaj, C.A. Balseiro, D. Feinberg, and M. Avignon, Phys. Rev. B 86, 214519 (2012).

44 T. Yokoyama, M. Eto, and Yu.V. Nazarov, J. Phys. Soc. Jpn. 82, 054703 (2013).

45 E. Goldobin, D. Koelle, R. Kleiner, and R.G. Mints, Phys. Rev. Lett. 107, 227001 (2011).

${ }^{46}$ V. Braude and Yu.V. Nazarov, Phys. Rev. Lett. 98, 077003 (2007).

47 J.F. Liu and K.S. Chan, Phys. Rev. B 82, 184533 (2010).

48 P.M.R. Brydon, C. Iniotakis, D. Manske, and M. Sigrist, Phys. Rev. Lett. 104, 197001 (2010).

49 M. Alidoust and J. Linder, Phys. Rev. B 87, 060503(R) (2013).

50 H. Sickinger, A. Lipman, M. Weides, R.G. Mints, H. Kohlstedt, D. Koelle, R. Kleiner, and E. Goldobin, Phys. Rev. Lett. 109, 107002 (2012).

51 V.B. Geshkenbein and A.I. Larkin, Pis'ma Zh. Eksp. Teor. Fiz. 43, 306 (1986) [JETP Lett. 43, 395 (1986)].

52 S. Yip, Phys. Rev. B 52, 3087 (1995).

${ }^{53}$ Y. Tanaka and S. Kashiwaya, Phys. Rev. B 56, 892 (1997).

54 Y. Asano, Y. Tanaka, M. Sigrist, and S. Kashiwaya, Phys. Rev. B 71, 214501 (2005).
55 Y. Avishai and T.K. Ng, Phys. Rev. B 81, 104501 (2010).

56 T. Novotný, A. Rossini, and K. Flensberg, Phys. Rev. B 72, 224502 (2005).

57 A. Schulz, A. Zazunov, and R. Egger, Phys. Rev. B 79, 184517 (2009).

58 M.S. Choi, C. Bruder, and D. Loss, Phys. Rev. B 62, 13569 (2000).

59 R. Zitko, M. Lee, R. López, R. Aguado, and M.S. Choi, Phys. Rev. Lett. 105, 116803 (2010).

60 C. Karrasch, S. Andergassen, and V. Meden, Phys. Rev. B 84, 134512 (2011).

61 S. Droste, S. Andergassen, and J. Splettstoesser, J. Phys.: Cond. Matt. 24, 415301 (2012).

62 M. Leijnse and K. Flensberg, Phys. Rev. B 86, 134528 (2012).

63 A.R. Wright and M. Veldhorst, Phys. Rev. Lett. 111, 096801 (2013).

64 I.C. Fulga, A. Haim, A.R. Akhmerov, and Y. Oreg, New J. Phys. 15045020 (2013).

65 Yu.V. Nazarov and Ya.M. Blanter, Quantum Transport: Introduction to Nanoscience (Cambridge University Press, 2009).

66 Within the wide-band approximation, different $\xi_{\mathbf{k}}$ in the two leads change only the respective density of states, which is taken into account in the $\Gamma^{(L, R)}$ matrices in Eq. (2.14). Similarly, no qualitative modifications of the physics arise for different BCS gaps, $\Delta_{L} \neq \Delta_{R}$, except for an overall decrease of the supercurrent.

67 A.A. Abrikosov, L.P. Gorkov, and I.E. Dzyaloshinksi, Methods of Quantum Field Theory in Statistical Physics (Prentice-Hall, Inc., Englewood Cliffs, New Jersey, 1963).

68 Perturbation theory may still fail when the excitation energy $\epsilon_{b}$ entering Eq. (3.11) is close to zero (i.e., smaller than the hybridization scales). For instance, in Eq. (3.11) with $i=1$, this causes a divergence of the supercurrents $I_{a, 0} \propto(\Gamma / \Delta)^{2}\left(\Delta / \epsilon_{b}\right) \rightarrow \infty$. In such a case, the addition (or removal) of a single Cooper pair to (from) the dot leaves the ground-state energy unchanged. This may happen when the single-particle energy differences are much smaller than $\Delta$ and we have large $E_{c}$ with $N_{0}=n_{g} \pm 1$.

69 The double-frequency integrals in Eq. 3.11 can be carried out analytically by replacing $f(\tau) \rightarrow \tilde{f}(\tau)$ in Eq. 2.15), with $\left(\omega^{2}+\Delta^{2}\right)^{-1 / 2} \rightarrow\left(\tilde{\Delta}^{2} / \Delta\right) /\left(\omega^{2}+\tilde{\Delta}^{2}\right)$, where $\tilde{\Delta}$ is a fitting parameter of order $\Delta$ chosen such that $f(\tau)$ and $\tilde{f}(\tau)$ have the same low-energy behavior. Within $1 \%$ accuracy, analytical results (using $\tilde{f}$ ) were found to match numerics (using $f$ ) for all parameters studied. We have used $f \rightarrow \tilde{f}$ exclusively for generating the figures in Sec. IV

70 Note that for $N_{0}=1$, the ground-state is two-fold spin degenerate. Since both spin orientations lead to the same result, our derivation (assuming non-degenerate $|G\rangle$ ) still holds.

71 Note that for $\epsilon \rightarrow$ 0, Eq. (4.2) coincides with Eq. (4.6). 